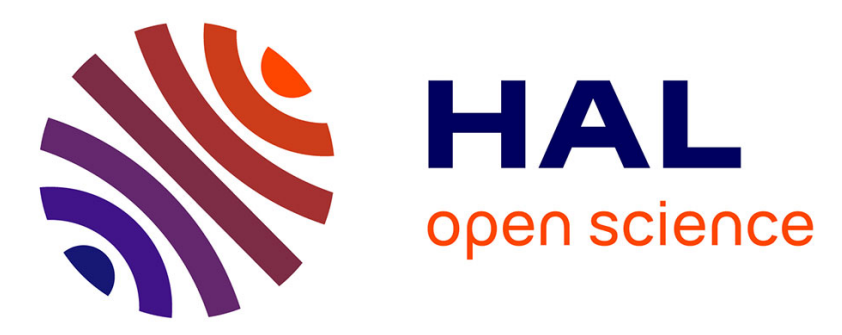

\title{
On the stationary macroscopic inertial effects for one phase flow in ordered and disordered porous media
}

Didier Lasseux, Ali Akbar Abbasian Arani, Azita Ahmadi-Senichault

\section{To cite this version:}

Didier Lasseux, Ali Akbar Abbasian Arani, Azita Ahmadi-Senichault. On the stationary macroscopic inertial effects for one phase flow in ordered and disordered porous media. Physics of Fluids, 2011, 23 (7), 19 p. 10.1063/1.3615514 . hal-01174068

\section{HAL Id: hal-01174068 \\ https://hal.science/hal-01174068}

Submitted on 8 Sep 2015

HAL is a multi-disciplinary open access archive for the deposit and dissemination of scientific research documents, whether they are published or not. The documents may come from teaching and research institutions in France or abroad, or from public or private research centers.
L'archive ouverte pluridisciplinaire HAL, est destinée au dépôt et à la diffusion de documents scientifiques de niveau recherche, publiés ou non, émanant des établissements d'enseignement et de recherche français ou étrangers, des laboratoires publics ou privés. 


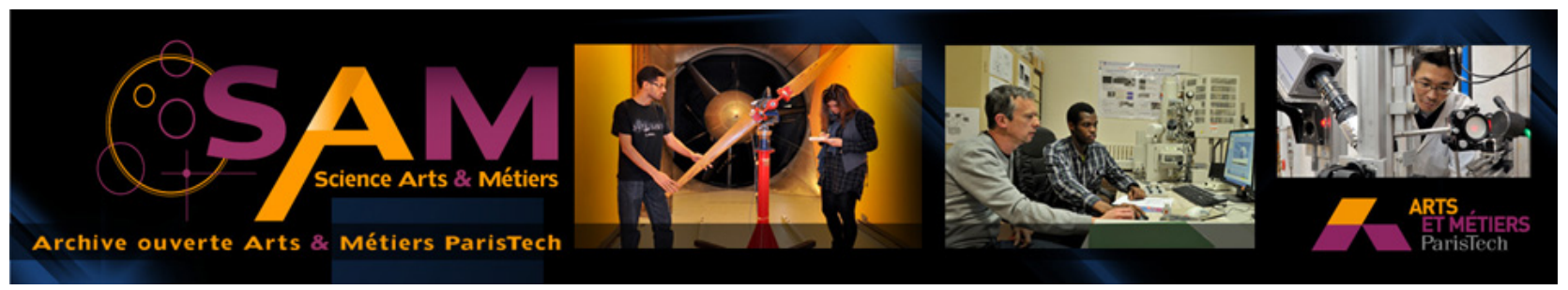

Science Arts \& Métiers (SAM)

is an open access repository that collects the work of Arts et Métiers ParisTech researchers and makes it freely available over the web where possible.

This is an author-deposited version published in: http://sam.ensam.eu

Handle ID: .http://hdl.handle.net/10985/9726

\section{To cite this version :}

Didier LASSEUX, Ali Akbar ABBASIAN ARANI, Azita AHMADI-SENICHAULT - On the stationary macroscopic inertial effects for one phase flow in ordered and disordered porous media - Physics of Fluids - Vol. 23, n7, p.19 p. - 2011 


\title{
On the stationary macroscopic inertial effects for one phase flow in ordered and disordered porous media
}

\author{
D. Lasseux, ${ }^{\text {a) }}$ A. A. Abbasian Arani, and A. Ahmadi \\ Université Bordeaux I, Transferts, Ecoulements, Fluides, Energétique (UMR CNRS 8508), Esplanade \\ des Arts et Métiers, 33405 Talence Cedex, France
}

\begin{abstract}
We report on the controversial dependence of the inertial correction to Darcy's law upon the filtration velocity (or Reynolds number) for one-phase Newtonian incompressible flow in model porous media. Our analysis is performed on the basis of an upscaled form of the Navier-Stokes equation requiring the solution of both the micro-scale flow and the associated closure problem. It is carried out with a special focus on the different regimes of inertia (weak and strong inertia) and the crossover between these regimes versus flow orientation and structural parameters, namely porosity and disorder. For ordered structures, it is shown that (i) the tensor involved in the expression of the correction is generally not symmetric, despite the isotropic feature of the permeability tensor. This is in accordance with the fact that the extra force due to inertia exerted on the structure is not pure drag in the general case; (ii) the Forchheimer type of correction (which strictly depends on the square of the filtration velocity) is an approximation that does not hold at all for particular orientations of the pressure gradient with respect to the axes of the structure; and (iii) the weak inertia regime always exists as predicted by theoretical developments. When structural disorder is introduced, this work shows that (i) the quadratic dependence of the correction upon the filtration velocity is very robust over a wide range of the Reynolds number in the strong inertia regime; (ii) the Reynolds number interval corresponding to weak inertia, that is always present, is strongly reduced in comparison to ordered structures. In conjunction with its relatively small magnitude, it explains why this weak inertia regime is most of the time overlooked during experiments on natural media. In all cases, the Forchheimer correction implies that the permeability is different from the intrinsic one. (C) 2011 American Institute of Physics.
\end{abstract} [doi:10.1063/1.3615514]

\section{INTRODUCTION}

It is now well established that the classical Darcy's law used to describe incompressible one phase Newtonian flow of a fluid $\beta$ in a homogeneous non-deformable porous medium and given by

$$
\left\langle\mathbf{v}_{\beta}\right\rangle=-\frac{\mathbf{K}}{\mu_{\beta}} \cdot\left(\nabla\left\langle p_{\beta}\right\rangle^{\beta}-\rho_{\beta} \mathbf{g}\right),
$$

$\left\langle\mathbf{v}_{\beta}\right\rangle$ being the seepage or Darcy velocity, $\nabla\left\langle p_{\beta}\right\rangle^{\beta}$ the macroscopic pressure gradient, $\mathbf{g}$ the gravitational acceleration, and $\mathbf{K}$ the permeability tensor, only holds when the porescale flow occurs in the creeping regime. The equivalent macroscopic constraint is that the Reynolds number, usually defined as

$$
\operatorname{Re}_{d}=\frac{\rho_{\beta}\left|\left\langle\mathbf{v}_{\beta}\right\rangle\right| d}{\mu_{\beta}},
$$

$d$ being a typical grain size of the porous medium, remains small compared to unity. Here we have used $\mu_{\beta}$ and $\rho_{\beta}$ to represent the dynamic viscosity and density of the $\beta$-phase and $\mathbf{g}$ for the gravitational acceleration. Originally obtained from experiments ${ }^{1}$ and later formalized using upscaling techniques, ${ }^{2,3}$ Eq. (1) must be reconsidered when inertial effects

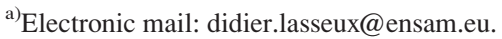

become significant. Active research has been dedicated to derive adequate corrections to the linear relationship in Eq. (1) from numerical, theoretical, and experimental points of view since the early work of Forchheimer ${ }^{4}$ where ad hoc 1D relationships of the form,

$$
\frac{\partial\left\langle p_{\beta}\right\rangle^{\beta}}{\partial x}=-\alpha\left\langle v_{\beta}\right\rangle-\rho_{\beta} \beta\left\langle v_{\beta}\right\rangle^{m}
$$

or

$$
\frac{\partial\left\langle p_{\beta}\right\rangle^{\beta}}{\partial x}=-\alpha\left\langle v_{\beta}\right\rangle-\rho_{\beta} \beta\left\langle v_{\beta}\right\rangle^{2}-\rho_{\beta}^{2} \gamma\left\langle v_{\beta}\right\rangle^{3},
$$

obtained on an empirical basis were proposed. ${ }^{5}$ While $\alpha, \beta$, and $\gamma$ were considered as parameters intrinsic to the medium, the exponent $m$ was identified to a value close to 2 confirming the popular Forchheimer form for Eq. (3). ${ }^{6-8}$ This popular form has been considered as a valid one, either from comparison to experimental data, ${ }^{9-13}$ theoretical derivation, ${ }^{14-21}$ or computational results. ${ }^{22-28}$ It was considered as a relevant local model for numerical upscaling in heterogeneous media. ${ }^{29}$ It has been extensively used in petroleum and chemical engineering applications for several decades. ${ }^{30-32}$ Deviation from Eq. (1), starting at $R e_{d}$ between 1 and 15 as observed by almost all authors, ${ }^{33-35}$ has been attributed to turbulence ${ }^{36}$ until the late 1960s. The physical 
justification of the quadratic nature of the correction was supported either by intuition or by dimensional analysis and the analogous turbulent kinetic energy loss in straight tubes. After a classification of flow regimes indicating that turbulence was not the origin of the deviation from the linearity of Eq. (1) ${ }^{37}$ experimental and unsteady numerical simulation evidence of the onset of turbulence for $R e_{d}$ on the order of 100 was demonstrated. ${ }^{35,38,39}$

During the early 1990s, a careful attention to the onset of deviation from Eq. (1) was reported in both numerical and theoretical works, questioning the quadratic dependence of the correction. Numerical simulation results obtained on model structures made of ordered arrays of cylinders of circular cross section lead to $m=3$ in (3). ${ }^{40}$ This result was confirmed independently from a theoretical point of view using double scale homogenization ${ }^{41-45}$ for Reynolds numbers (based on the characteristic pore dimension) in the range $\left[\delta^{1 / 2}, 1\right]$ where $\delta$ is the scale separation parameter, i.e., the ratio of the microscopic to macroscopic characteristic scales. This so-called weak inertia regime was first obtained in the case of periodic homogeneous and isotropic media but was extended to the case of anisotropic ones with the restriction of invariance while reversing the flow direction, ${ }^{46,47}$ although this last constraint was further relaxed shortly after. ${ }^{48}$ This regime was also evidenced for flow in a rough fracture. ${ }^{49}$ Further numerical simulations confirmed this result $^{39,50-52}$ and lead to a classification of the non-linear deviation from Darcy's law involving, at least, three different regimes separated by transitions: ${ }^{48}$ (i) the weak inertia regime which appears at the onset of non-linearity and where the correction term scales as $\left\langle v_{\beta}\right\rangle^{3}$ for Reynolds numbers based on the characteristic pore dimension in the interval $\left[\delta^{1 / 2}, 1\right]$, (ii) a strong inertia regime where the correction is quadratic in $\left\langle v_{\beta}\right\rangle$, i.e., a Forchheimer type of correction, for the Reynolds number $\gtrsim 1$ to 10 , and (iii) turbulence which, will not be discussed further in the present work, for the Reynolds number $\gtrsim 100$.

Precise physical justification of these cubic and quadratic dependences are still a matter of questioning. ${ }^{44}$ Since macroscopic inertial forces are clearly weak compared to viscous ones, ${ }^{18,23,53}$ non-linearity in the drag-velocity relationship must originate from microscopic inertial and viscous forces. Invoking the former as the dominant effect ${ }^{54,55}$ is justified by (i) streamlines deformation resulting from bends in flow paths (i.e., tortuosity) ${ }^{56}$ and in constrictions and enlargements, (ii) backflow as well as flow separation yielding form drag, (iii) fluid flow channeling which gradually disappears with increasing velocity leading to a strong modification of kinetic energy loss distribution within the medium. ${ }^{57}$ Non-linearity resulting from viscous drag can be justified by the development of boundary layers at pore walls as observed experimentally ${ }^{35}$ giving rise to inertial core flow at the pore scale. Due to boundary layers growing with increasing Reynolds numbers, local velocity in the cores increases non-linearly. Coupling between inertial and viscous effects must also be considered with the dissipation in recirculation zones. ${ }^{24}$ This coupling has recently led to propose a correction to Eq. (1) in a complex empirical exponential form rather than a polynomial one. ${ }^{58}$
Even if the flow classification under the Darcy, weak and strong inertia regimes have been widely admitted, the dependence of the transition between these different regimes, when they can be identified, upon structural properties of the medium such as porosity, disorder, and anisotropy has not been yet documented. Moreover, as shown in many theoretical developments, ${ }^{18,20,41,42,53,59}$ the non-linear correction to Eq. (1) involves a correction tensor. Nevertheless, all reported numerical (and experimental) analyses of this correction are performed on the velocity magnitude using a scalar only. Our purpose in this work is to shed light on these two aspects of the problem that are of practical major importance. On the one hand, inspecting the tensorial form of the macroscopic inertial resistance is not only useful to analyze the anisotropic character of the non-Darcy part of the flow but is required in the perspective of a second upscaling over a heterogeneous medium involving different regions, each of them being characterized by its non-Darcy correction (and permeability) tensors. On the other hand, analyzing the dependence of the different regimes, when they can be reasonably distinguished, as well as the transition between them upon structural parameters of the medium is also necessary to help understanding the physical origin of these regimes and refine the macroscopic description of the flow. This opens the way for many other investigations on anisotropic and heterogeneous media as well as on inertial two-phase flow as already explored in preliminary works. ${ }^{60,61}$

Since analytical solutions to the Navier-Stokes problem are restricted to simple geometries, at asymptotically small Reynolds numbers and for ordered structures having very large or exceedingly small porosities, most of the time making use of the Oseen's approximation, we chose to perform comprehensive direct numerical simulations. They were performed in the Reynolds ranges 0-150 (ordered arrays) and 0-30 (disordered arrays) and for porosities between 0.3 and 0.75 . The analysis is performed on the basis of a theoretical derivation of the macroscopic mass and momentum equations obtained by volume averaging the incompressible onephase Navier-Stokes problem..$^{53}$ Ordered media are of major interest for these synthetic structures are specifically designed for many microfluidics devices whereas disorder is inherent to natural porous structures like geological materials.

The paper is organized as follows. In Sec. II, the macroscopic model and the associated closure problems yielding both the permeability and correction tensors are reported. After presenting the numerical method used to compute the two tensors (its validation is provided in Appendix A), results are discussed in Sec. III. Computations were carried out with special attention to high accuracy in order to investigate the existence of the different regimes, the scaling laws in these regimes as well as the transition (or crossover) between them versus flow orientation and structural parameters, namely porosity and disorder. This was performed on 2D model structures of a porous medium corresponding to ordered, weakly and strongly disordered arrays of parallel cylinders of square cross sections. Important concluding remarks are reported in Sec. IV. 


\section{GOVERNING EQUATIONS AND SOLUTION}

\section{A. Microscopic boundary value problem and macroscopic model}

The single-phase flow of an incompressible Newtonian fluid $\beta$ is considered in a macroscopic region of a rigid porous medium. The boundary value problem describing the process at the microscopic (pore) scale is given by the classical mass and momentum (Navier-Stokes) balance equations,

$$
\begin{gathered}
\rho_{\beta}\left(\frac{\partial \mathbf{v}_{\beta}}{\partial t}+\mathbf{v}_{\beta} \cdot \nabla \mathbf{v}_{\beta}\right)=-\nabla p_{\beta}+\rho_{\beta} \mathbf{g}+\mu_{\beta} \nabla^{2} \mathbf{v}_{\beta}, \\
\nabla \cdot \mathbf{v}_{\beta}=0
\end{gathered}
$$

with the boundary conditions,

$$
\begin{gathered}
\mathbf{v}_{\beta}=0 \quad \text { at } A_{\beta \sigma}, \\
\mathbf{v}_{\beta}=\phi(\mathbf{r}, t) \quad \text { at } A_{\beta e} .
\end{gathered}
$$

In these equations $\mathbf{v}_{\beta}$ and $p_{\beta}$ are, respectively, the velocity and pressure of the $\beta$-phase; $A_{\beta \sigma}$ represents the interface area between the $\beta$-phase and the solid phase $\sigma$ contained within the macroscopic region, while $A_{\beta e}$ represents the $\beta$ phase entrances and exits of the macroscopic region on which the velocity is defined by $\phi(\mathbf{r}, t)$.

Upscaling of this problem was proposed using double scale homogenization $^{41,42}$ under the restriction of very small Reynolds numbers based on the characteristic pore size. More recently, it was developed using the volume averaging method. ${ }^{53}$ In this latter work, it was demonstrated that the macroscopic model is given by

$$
\begin{gathered}
\nabla \cdot\left\langle\mathbf{v}_{\beta}\right\rangle=0, \\
\left\langle\mathbf{v}_{\beta}\right\rangle=\frac{-\mathbf{K}}{\mu_{\beta}} \cdot\left(\nabla\left\langle p_{\beta}\right\rangle^{\beta}-\rho_{\beta} \mathbf{g}\right)-\mathbf{F} \cdot\left\langle\mathbf{v}_{\beta}\right\rangle,
\end{gathered}
$$

where $\mathbf{K}$ (having the dimension of $m^{2}$ ) and $\mathbf{F}$ (dimensionless) are the permeability and Darcy's law correction tensors, respectively, and in which the following definitions of the superficial and intrinsic phase averages of any quantity $\psi_{\beta}$ associated to the $\beta$-phase are respectively given by

$$
\left\langle\psi_{\beta}\right\rangle=\frac{1}{V} \int_{V_{\beta}} \psi_{\beta} d V
$$

and

$$
\left\langle\psi_{\beta}\right\rangle^{\beta}=\varepsilon^{-1}\left\langle\psi_{\beta}\right\rangle=\frac{1}{V_{\beta}} \int_{V_{\beta}} \psi_{\beta} d V .
$$

In these two last relationships, $V_{\beta}$ represents the volume of the $\beta$-phase contained within the averaging volume, $V$ at least as large as the Representative Elementary Volume (REV) of the structure and

$$
\varepsilon=\frac{V_{\beta}}{V}
$$

is the porosity of the medium.
The macroscopic model in Eqs. (9) and (10) remains valid provided two constraints are satisfied. First, scale hierarchy must be such that

$$
l_{\beta} \ll r_{0} \ll L
$$

where $l_{\beta}$ is the characteristic length-scale in the $\beta$-phase (pore diameter), $r_{0}$ is the radius of the averaging volume, and $L$ is the macroscopic length-scale. Second the characteristic time $t^{*}$ at which the process is observed must satisfy

$$
\frac{\mu_{\beta} t^{*}}{\rho_{\beta} l_{\beta}^{2}} \gg 1
$$

or equivalently

$$
\frac{\rho_{\beta}\left|\left\langle\mathbf{v}_{\beta}\right\rangle^{\beta}\right| l_{\beta}}{\mu_{\beta}}\left(l_{\beta} / L\right) \ll 1,
$$

for the quasi-steady nature of the macroscopic flow to hold. Under these circumstances and within the framework of a spatially periodic model of a porous medium, $\mathbf{K}$ and $\mathbf{F}$ can be explicitly determined from the solution over the unit cell of the periodic structure (period $l_{i}, i=1,2,3$ ) of two closure problems respectively given by ${ }^{53,62,63}$

$$
\begin{aligned}
& \left\{\begin{array}{c}
0=-\nabla \mathbf{d}+\nabla^{2} \mathbf{D}+\mathbf{I} \\
\nabla \cdot \mathbf{D}=0 \\
\mathbf{D}=0 \quad \text { at } \quad A_{\beta \sigma} \\
\mathbf{d}\left(\mathbf{r}+l_{i}\right)=\mathbf{d}(\mathbf{r}) \mathbf{D}\left(\mathbf{r}+l_{i}\right)=\mathbf{D}(\mathbf{r}) i=1,2,3
\end{array}\right. \\
& \langle\mathbf{D}\rangle=\mathbf{K} \\
& \left\{\begin{array}{c}
\frac{\rho_{\beta} \mathbf{v}_{\beta}}{\mu_{\beta}} \cdot \nabla \mathbf{M}=-\nabla \mathbf{m}+\nabla^{2} \mathbf{M}+\mathbf{I} \\
\nabla \cdot \mathbf{M}=0 \\
\mathbf{M}=0 \quad \text { at } \quad A_{\beta \sigma} \\
\mathbf{m}\left(\mathbf{r}+l_{i}\right)=\mathbf{m}(\mathbf{r}) \mathbf{M}\left(\mathbf{r}+l_{i}\right)=\mathbf{M}(\mathbf{r}) i=1,2,3
\end{array}\right. \\
& \langle\mathbf{M}\rangle=\mathbf{H}
\end{aligned}
$$

while

$$
\mathbf{F}=\mathbf{K} \cdot \mathbf{H}^{-1}-\mathbf{I}
$$

Here, $\mathbf{H}$ is a flow-dependent tensor and corresponds to the apparent permeability ${ }^{64}$ allowing an alternate form of Eq. (10),

$$
\left\langle\mathbf{v}_{\beta}\right\rangle=\frac{-\mathbf{H}}{\mu_{\beta}} \cdot\left(\nabla\left\langle p_{\beta}\right\rangle^{\beta}-\rho_{\beta} \mathbf{g}\right) .
$$

Important remarks must be made at this point. First, it must be emphasized that under the assumptions of well separated scales (relation (14)), steady flow (relation (15)) and for a spatially periodic medium, the determination of F obtained from the solution of Eqs. (17) through (21) is exact regardless of the Reynolds number value. ${ }^{53}$ In addition, while $\mathbf{K}$ can be shown to be symmetric (and positive definite), ${ }^{65} \mathbf{F}$ and $\mathbf{H}$ are not, as will be proved further with our numerical results. Finally, it must be stressed that, while $\mathbf{K}$ is a quantity intrinsic to the structure, $\mathbf{F}$ and $\mathbf{H}$ 
depend not only on the medium but also on both the Reynolds number and pressure gradient orientation. As a consequence, the macroscopic force resulting from flow in an isotropic unit cell is no longer pure drag as it would be in the Darcy regime for any orientation of the flow. Exception is when the isotropic unit cell possesses at least one symmetry axis and flow is aligned with this axis. Evidence of these features is shown below.

\section{B. Determination of the macroscopic properties}

Without losing generality, gravity is not considered in the rest of this work. While $\mathbf{K}$ can be determined either directly from the solution of the creeping flow over a periodic unit cell or equivalently from the closure problem (17), the determination of $\mathbf{H}$ (or $\mathbf{F}$ ) requires the solution of the stationary Navier-Stokes flow problem over the unit cell. Once dimensionless variables denoted by the superscript* given by

$$
\begin{gathered}
x^{*}=\frac{x}{l} \quad y^{*}=\frac{y}{l} \quad z^{*}=\frac{z}{l}, \\
\mathbf{v}_{\beta}^{*}=\frac{\mathbf{v}_{\beta}}{v_{r e f}} \quad p_{\beta}^{*}=\frac{p_{\beta}}{p_{r e f}}
\end{gathered}
$$

are used along with the decomposition, ${ }^{66,67}$

$$
p_{\beta}=\left\langle p_{\beta}\right\rangle^{\beta}+\tilde{p}_{\beta},
$$

the cellular flow problem can be written as (for simplicity, the superscript $*$ is omitted on the $\nabla$ operator),

$$
\begin{gathered}
R e^{*} \mathbf{v}_{\beta}^{*} \cdot \nabla \mathbf{v}_{\beta}^{*}+\nabla \tilde{p}_{\beta}^{*}-\nabla^{2} \mathbf{v}_{\beta}^{*}=-\nabla\left\langle p_{\beta}^{*}\right\rangle^{\beta}, \\
\nabla \cdot \mathbf{v}_{\beta}^{*}=0, \\
\mathbf{v}_{\beta}^{*}=0 \quad \text { at } A_{\beta \sigma}, \\
\mathbf{v}_{\beta}^{*}\left(\mathbf{r}^{*}+l_{i}^{*}\right)=\mathbf{v}_{\beta}^{*}\left(\mathbf{r}^{*}\right) \tilde{p}_{\beta}^{*}\left(\mathbf{r}^{*}+l_{i}^{*}\right)=\tilde{p}_{\beta}^{*}\left(\mathbf{r}^{*}\right) i=1,2,3 .
\end{gathered}
$$

In Eq. (23), $l$ represents one of the edge sizes of either the periodic unit cell in the ordered case or the random cell when disorder is introduced (see Figures 1(b) and 14) while in Eq. (26), $R e^{*}$ is a Reynolds number defined by

$$
R e^{*}=\frac{\rho_{\beta} v_{r e f} l}{\mu_{\beta}} .
$$

A suitable choice of the reference velocity, $v_{r e f}$, and pressure, $p_{\text {ref }}$, is

$$
v_{r e f}=\frac{l^{2}}{\mu_{\beta}}\left|\nabla\left\langle p_{\beta}\right\rangle^{\beta}\right| \quad p_{r e f}=l\left|\nabla\left\langle p_{\beta}\right\rangle^{\beta}\right|
$$

Since $\left|\nabla\left\langle p_{\beta}^{*}\right\rangle^{\beta}\right|=1$, the original choice of the intensity and orientation of the external force per unit volume, $\nabla\left\langle p_{\beta}\right\rangle^{\beta}$, applied on the periodic structure is now done by selecting a $R e^{*}$ and the orientation of $\nabla\left\langle p_{\beta}^{*}\right\rangle^{\beta}$. As indicated above, solving the problem (26) through (29) for $R e^{*}=0$ and $\nabla\left\langle p_{\beta}^{*}\right\rangle^{\beta}$

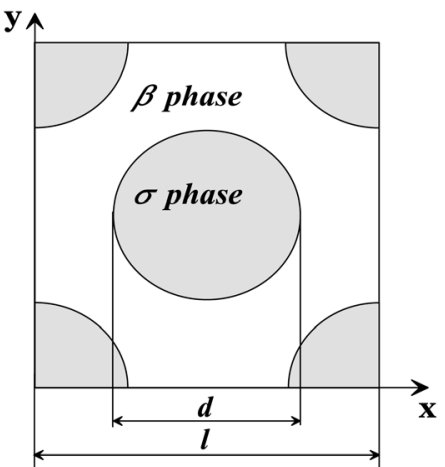

a)

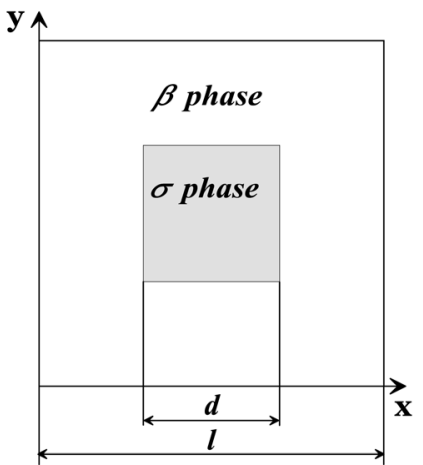

b)
FIG. 1. Unit cell (a) used for validation tests and $b$ ) of the ordered structure used to compute $\mathbf{F}$.

successively along the three directions $\left(\mathbf{e}_{\mathbf{x}}, \mathbf{e}_{\mathbf{y}}, \mathbf{e}_{\mathbf{z}}\right)$ of periodicity axes of the unit cell is equivalent to solving the closure problem (17) and yields $\mathbf{K}^{*}=\mathbf{K} / l^{2}$ since in this flow regime,

$$
\left\langle\mathbf{v}_{\beta}^{*}\right\rangle=-\mathbf{K}^{*} \cdot \nabla\left\langle p_{\beta}^{*}\right\rangle^{\beta} .
$$

For a given $R e^{*} \neq 0$ and orientation of $\nabla\left\langle p_{\beta}^{*}\right\rangle^{\beta}$, the determination of $\mathbf{H}^{*}=\mathbf{H} / l^{2}$ (or $\mathbf{F}^{*}=\mathbf{F}$ ) is more computational time consuming. It is performed by solving first the periodic stationary Navier-Stokes problem (26) through (29) to obtain $\mathbf{v}_{\beta}^{*}$. In a second step, this result is inserted in the dimensionless form of the closure problem (19), namely

$$
\left\{\begin{array}{c}
R e^{*} \mathbf{v}_{\beta}^{*} \cdot \nabla \mathbf{M}^{*}+\nabla \mathbf{m}^{*}-\nabla^{2} \mathbf{M}^{*}=\mathbf{I} \\
\nabla \cdot \mathbf{M}^{*}=0 \\
\mathbf{M}^{*}=0 \quad \text { at } \quad A_{\beta \sigma} \\
\mathbf{m}^{*}\left(\mathbf{r}^{*}+l_{i}^{*}\right)=\mathbf{m}^{*}\left(\mathbf{r}^{*}\right) \mathbf{M}^{*}\left(\mathbf{r}^{*}+l_{i}^{*}\right)=\mathbf{M}^{*}\left(\mathbf{r}^{*}\right) \quad i=1,2,3
\end{array},\right.
$$

yielding $\mathbf{H}^{*}$ and $\mathbf{F}$ according to

$$
\begin{gathered}
\left\langle\mathbf{M}^{*}\right\rangle=\mathbf{H}^{*}, \\
\mathbf{F}=\mathbf{K}^{*} \cdot \mathbf{H}^{*-1}-\mathbf{I} .
\end{gathered}
$$

This problem has again a Navier-Stokes structure and can be solved with the same procedure as the one used to solve the flow. With all these considerations, the same Navier-Stokes solver can in fact be used to determine both $\mathbf{K}^{*}$ and $\mathbf{F}$.

In order to investigate the deviation from Darcy's law, we consider the macroscopic force per unit volume $\mathbf{f}$ exerted on the structure $\left(\mathbf{f}=-\mu_{\beta} \mathbf{H}^{-1} \cdot\left\langle\mathbf{v}_{\beta}\right\rangle\right)$ and decompose this force into the Darcy part $\left(\mathbf{f}_{\mathbf{d}}=-\mu_{\beta} \mathbf{K}^{-1} \cdot\left\langle\mathbf{v}_{\beta}\right\rangle\right)$ and the contribution $\mathbf{f}_{\mathbf{i}}$ from inertia $\left(\mathbf{f}_{\mathbf{i}}=-\mu_{\beta} \mathbf{K}^{-1} \cdot \mathbf{F} \cdot\left\langle\mathbf{v}_{\beta}\right\rangle\right)$. Classically, in many references as in Koch and Ladd, ${ }^{39}$ analysis is based on the modulus of $\frac{l^{2}}{\mu_{\beta}\left|\left\langle\mathbf{v}_{\beta}\right\rangle\right|} \mathbf{f}=\frac{-\mathbf{K}^{*-1} \cdot(\mathbf{F}+\mathbf{I}) \cdot\left\langle\mathbf{v}_{\beta}^{*}\right\rangle}{\left|\left\langle\mathbf{v}_{\beta}^{*}\right\rangle\right|}$, i.e., on $\frac{1}{\left|\left\langle\mathbf{v}_{\beta}^{*}\right\rangle\right|}$. An alternative is to focus on a normalized form, $\mathbf{f}_{\mathbf{c}}$, of $\mathbf{f}_{\mathbf{i}}$ using $\mu_{\beta}\left|\left\langle\mathbf{v}_{\beta}\right\rangle\right| \mathbf{K}^{-1}$ as the scaling factor which yields a unit normalized vector for $\mathbf{f}_{\mathbf{d}}$. Hence, in the sequel of this work, flow regimes are analyzed in terms of the dimensionless correction vector $\mathbf{f}_{\mathbf{c}}$ given by 


$$
\mathbf{f}_{\mathbf{c}}=\frac{\mathbf{K}}{\mu_{\beta}\left|\left\langle\mathbf{v}_{\beta}\right\rangle\right|} \cdot \mathbf{f}_{\mathbf{i}}=\frac{-\mathbf{F} \cdot\left\langle\mathbf{v}_{\beta}\right\rangle}{\left|\left\langle\mathbf{v}_{\beta}\right\rangle\right|}=\frac{-\mathbf{F} \cdot\left\langle\mathbf{v}_{\beta}^{*}\right\rangle}{\left|\left\langle\mathbf{v}_{\beta}^{*}\right\rangle\right|}
$$

while $\left\langle\mathbf{v}_{\beta}^{*}\right\rangle=-\mathbf{H}^{*} \cdot \nabla\left\langle p_{\beta}^{*}\right\rangle^{\beta}=-\mathbf{K}^{*} \cdot \nabla\left\langle p_{\beta}^{*}\right\rangle^{\beta}-\mathbf{F} \cdot\left\langle\mathbf{v}_{\beta}^{*}\right\rangle$. Indeed, this form is more sensitive to the Reynolds number variations than the traditional one proposed in the literature. It should be noted that, if one is only interested in analyzing the correction, the resolution of the closure problem for $\mathbf{H}$ (or $\mathbf{F}$ ) is not required. In fact, one can solve the flow (Eqs. (26) through (29)) and compute $\left\langle\mathbf{v}_{\beta}^{*}\right\rangle$ to get $\mathbf{f}_{\mathbf{c}}$ from an equivalent form of Eq. (36),

$$
\mathbf{f}_{\mathbf{c}}=\frac{\left\langle\mathbf{v}_{\beta}^{*}\right\rangle+\mathbf{K}^{*} \cdot \nabla\left\langle p_{\beta}^{*}\right\rangle^{\beta}}{\left|\left\langle\mathbf{v}_{\beta}^{*}\right\rangle\right|} .
$$

Since the correction is analyzed through its dependence upon the Reynolds number, attention must also be dedicated to the definition of this number. In the following, we shall use two forms different from $R e^{*}$, namely $R e_{d}$ (see Eq. (2)) and $R e_{k}$, respectively given by $26,39,51$

$$
R e_{d}=\left|\left\langle\mathbf{v}_{\beta}^{*}\right\rangle\right| d^{*} R e^{*}=\frac{\rho_{\beta}\left|\left\langle\mathbf{v}_{\beta}\right\rangle\right| d}{\mu_{\beta}},
$$

where $d^{*}=d / l$ is the dimensionless grain size of the porous structure and

$$
R e_{k}=\left|\left\langle\mathbf{v}_{\beta}^{*}\right\rangle\right| \sqrt{k^{*}} R e^{*}=\frac{\rho_{\beta}\left|\left\langle\mathbf{v}_{\beta}\right\rangle\right| \sqrt{k}}{\mu_{\beta}} .
$$

When the structure is isotropic, i.e. $\mathbf{K}^{*}=k^{*} \mathbf{I},(\mathbf{K}=k \mathbf{I})$, the latter definition is preferable to the former and this is motivated by the fact that, from a practical point of view, $d$ is not necessarily well defined for all kinds of porous media and most of the time unknown a priori whereas $k$ is accessible by rather simple experiments. Moreover, $R e_{k}$ is scale-consistent since it involves macroscopic quantities only. As evidenced by Eqs. (36) and (39), a $\left|\left\langle\mathbf{v}_{\beta}\right\rangle\right|^{3}$ and $\left|\left\langle\mathbf{v}_{\beta}\right\rangle\right|^{2}$ dependence of $\mathbf{f}_{\mathbf{i}}$ in a weak and strong inertia regime respectively implies a corresponding $\operatorname{Re}_{k}^{2}$ and $R e_{k}$ dependence of $\mathbf{f}_{\mathbf{i}}$.

The closure problem (33) is solved using the numerical procedure described in the following section. The inertial correction is then analyzed using Eqs. (34) to (36) in the case of ordered, weakly and strongly disordered structures and is discussed in Sec. III.

\section{Method of resolution and validation}

To determine the two tensors $\mathbf{K}^{*}$ and $\mathbf{F}$ and the correction vector $\mathbf{f}_{\mathbf{c}}$, a $3 \mathrm{D}$ numerical procedure was developed on the basis of a finite volume formulation over a Marker And Cell (staggered) Cartesian regular grid. ${ }^{68}$ This technique is known to be both accurate and easy to implement leading to very conservative schemes. The viscous diffusive term was discretized with a second order centered scheme while the inertial convective term was discretized with a Quadratic Upwind Interpolation for Convective Kinematics (QUICK) scheme. This scheme, initially proposed by Leonard ${ }^{69}$ reveals to be very stable, fast converging and highly accurate avoiding significant numerical diffusion in comparison to hybrid schemes. ${ }^{70,71}$ Alternative forms of the QUICK discretization procedure were proposed in the literature ${ }^{72,73}$ and, in the present work, we used an improved version of this scheme. ${ }^{74}$ Velocity and pressure fields were sought iteratively following an artificial compressibility algorithm. ${ }^{75}$ Starting from a guessed pressure field, this algorithm consists in computing the momentum equation separately from the continuity equation, this latter being substituted by a perturbed divergence equation. At each iteration of this algorithm, the linear non-symmetric system was solved using a bi-conjugate gradient (or conjugate gradient when $R e^{*}=0$ to compute $\mathbf{K}^{*}$ ) routine. Under- (or inertial) relaxation was used to improve convergence especially for the larger Reynolds numbers investigated in this study.

Since our goal is to analyze the correction tensor $\mathbf{F}$ in a quantitative manner, it is important to first check the validity and the accuracy of the numerical procedure described above. To do so, the unit cell of an ordered array of parallel solid cylinders of circular cross section having the same diameter and arranged on a square centered lattice was used (see Figure 1(a)). The case $\nabla\left\langle p_{\beta}^{*}\right\rangle^{\beta}=\mathbf{e}_{\mathbf{x}}$ was considered which, for evident reasons of isotropy and symmetry about $\mathbf{e}_{\mathbf{x}}$, leads to $\left\langle\mathbf{v}_{\beta}^{*}\right\rangle \cdot \mathbf{e}_{\mathbf{y}}=0$, a diagonal $\mathbf{F}$ tensor and a zero $y$ component of $\mathbf{f}_{\mathbf{c}}$. Consequently, this unique pressure gradient orientation yields $k^{*}\left(\mathbf{K}^{*}=k^{*} \mathbf{I}\right)$ when $R e^{*}=0$ and provides the normalized correction $f_{c x}$ to the velocity given in Eq. (36) which reduces to the first diagonal term $f_{x x}$ of $\mathbf{F}$. For comparison purposes, flow computations were performed with parameters used elsewhere, ${ }^{27}$ i.e., with $\varepsilon=0.386$ yielding $d^{*} \cong 0.62521$. Details of the comparison are provided in Appendix A. The excellent agreement of our results with this reference and some data obtained with a Boundary Element Method validates the numerical method. Considering, however, that accuracy of the finite volume method might suffer from geometrical representation of curved objects with Cartesian grids as well as from pure grid size effect, we choose, in the rest of this work, to analyze the behaviour of $\mathbf{f}_{\mathbf{c}}$ and $\mathbf{F}$ on $2 \mathrm{D}$ configurations involving square objects.

\section{RESULTS AND DISCUSSION}

In this section, the inertial correction to Darcy's law is investigated through extensive numerical simulations on 2D ordered and disordered structures.

\section{A. Ordered structure}

We start the analysis with the case of a $2 \mathrm{D}$ isotropic porous medium for which the unit cell is represented in Figure 1(b). Accuracy was first investigated in the case $\nabla\left\langle p_{\beta}^{*}\right\rangle^{\beta}=\mathbf{e}_{\mathbf{x}}$ with $\varepsilon=0.75$. As in the test case used for validation, this choice of the orientation of $\nabla\left\langle p_{\beta}^{*}\right\rangle^{\beta}$ allows the computation of $k^{*}$ and the non-Darcy correction which reduces again to $f_{c x}=f_{x x}$. Permeability was computed varying the number of grid blocks from 400 to 250000 with the 
same convergence criteria as those employed for the validation (see Appendix A). Results on $k^{*}$ along with the corresponding relative errors, using $k^{*}$ obtained with 250000 grid blocks as the reference value, are reported in Table I. They clearly show the excellent accuracy achieved even with relatively coarse grids.

On this basis, $f_{c x}\left(=f_{x x}\right)$ was computed on the same unit cell using four different grid sizes. The convergence criterion on the overall algorithm was taken as $\max \left(10^{-7} \times f_{x x}, 10^{-12}\right)$ on the $L^{2}$ norm of $\nabla \cdot\left\langle\mathbf{v}_{\beta}^{*}\right\rangle$ over all grid blocks at each value of the Reynolds number. The convergence criterion for the linear system solver was $10^{3}$ times smaller. Computations were performed for $10^{-3} \leq R e^{*} \leq 10^{4}$ (i.e., $1.5 \times 10^{-6} \leq R e_{d} \leq 60$ or $\left.6.5 \times 10^{-6} \leq R e_{k} \leq 13.6\right)$ assuming that the stationary laminar solution is still physically valid in the upper range of these Reynolds numbers. An example of pressure and velocity fields is depicted in Figure 2 and results on the variation of $f_{c x}$ versus $R e_{k}$ are represented in Figure 3.

For the two coarser grids investigated here, the dimensionless inertial correction, $\mathbf{f}_{c}$, seems to vary linearly with $R e_{k}$ at the onset of deviation from Darcy's law which means that the dimensional correction, $\mathbf{f}_{i}$, would have a quadratic dependence on the velocity (see Figure 3(b)). Excessive attention must be paid, however, to this phenomenon which is a pure grid size effect. In fact, when macroscopic properties are computed using $200 \times 200$ grid blocks, one clearly observes a weak inertia regime $\left(7.4 \times 10^{-4} \lesssim R e_{k} \lesssim 0.3\right.$; $3.2 \times 10^{-3} \lesssim R e_{d} \lesssim 1.3$ ) where $f_{c x}$ scales as $\operatorname{Re}_{k}^{2}$ (see Figures 3 and $4(\mathrm{a}))$ and a strong inertia regime $\left(0.9 \lesssim R e_{k} \lesssim 2.2\right.$; $\left.3.9 \lesssim R e_{d} \lesssim 9.6\right)$ where $f_{c x}$ varies linearly with $R e_{k}$ (see Figure 4(b)). In this particular configuration where the mean flow is basically $1 \mathrm{D}$, this last regime would allow a relationship between the pressure gradient and seepage velocity of the form reported in Eq. (3) where $\beta$ is the inertial resistance factor. The two regimes are separated by a transition (or crossover) region $\left(0.3 \lesssim R e_{k} \lesssim 0.9\right)$ the extent of which will be shortly discussed below. At sufficiently low Reynolds numbers $\left(R e_{k} \lesssim 7 \times 10^{-6} ; R e_{d} \lesssim 3 \times 10^{-5}\right)$ and for a finer grid size (i.e., $400 \times 400$ ), the Darcy regime is obviously recovered asymptotically. This leads to a classification of the different regimes (Darcy, weak, and strong inertia) in accordance with that proposed in the literature. ${ }^{48,51}$ For larger Reynolds numbers, assuming that the stationary NavierStokes model is still physically meaningful, another regime appears where $f_{c x}$ varies again with $R e_{k}^{2}$ (see Figure 4(c)). However, this last regime is beyond the scope of this paper

TABLE I. Permeability variation with the number of grid blocks. Unit cell of Figure 1(b) with $\varepsilon=0.75$.

\begin{tabular}{lcc}
\hline \hline No. of grid blocks & $\mathrm{k}^{*}$ & $\Delta \mathrm{k}^{*} / \mathrm{k}^{*} \%$ \\
\hline $20 \times 20$ & 0.0130765 & 0.4089 \\
$40 \times 40$ & 0.0130355 & 0.0941 \\
$120 \times 120$ & 0.0130242 & 0.0074 \\
$200 \times 200$ & 0.0130235 & 0.0018 \\
$400 \times 400$ & 0.0130233 & $7.810^{-5}$ \\
$500 \times 500$ & 0.0130232 & - \\
\hline \hline
\end{tabular}
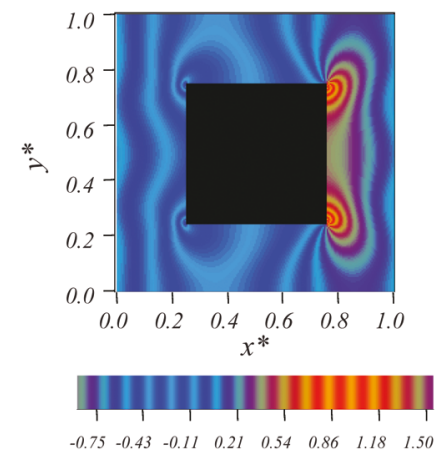

a)

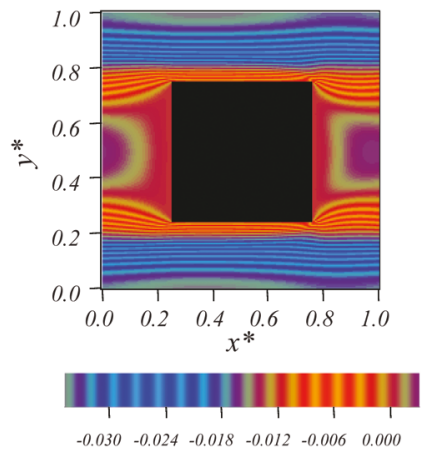

b)

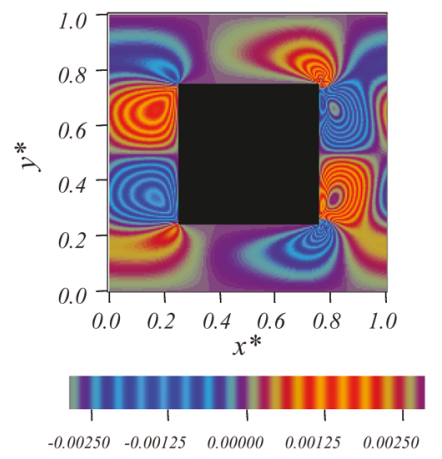

c)
FIG. 2. (Color) Numerical results obtained on the unit cell of figure 1(b), $\left.\varepsilon=0.75, \nabla\left\langle p_{\beta}^{*}\right\rangle=\mathbf{e}_{x}, \operatorname{Re}_{k}=13.6\left(R e_{d}=59.6\right), a\right) \tilde{p}_{\beta}^{*}$ (see Eq. (25)) and (b) $\left.\mathbf{v}_{\beta}^{*} \cdot \mathbf{e}_{\mathbf{x}}, c\right) \mathbf{v}_{\beta}^{*} \cdot \mathbf{e}_{\mathbf{y}}$.

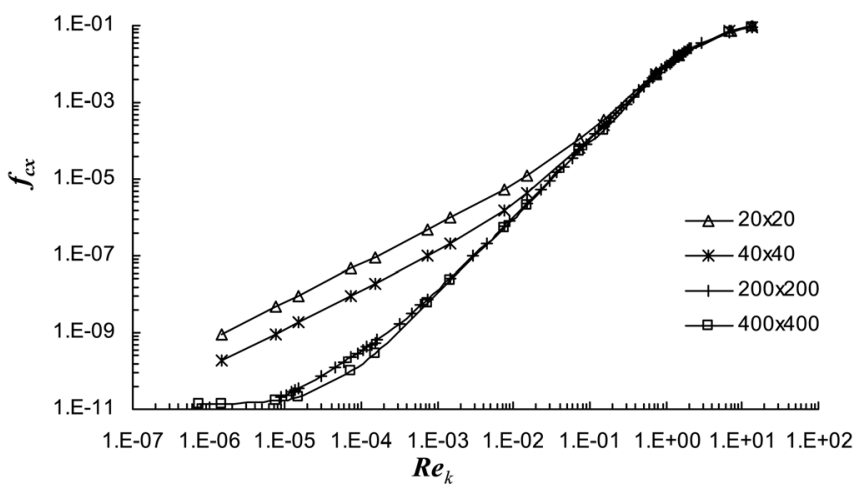

a)

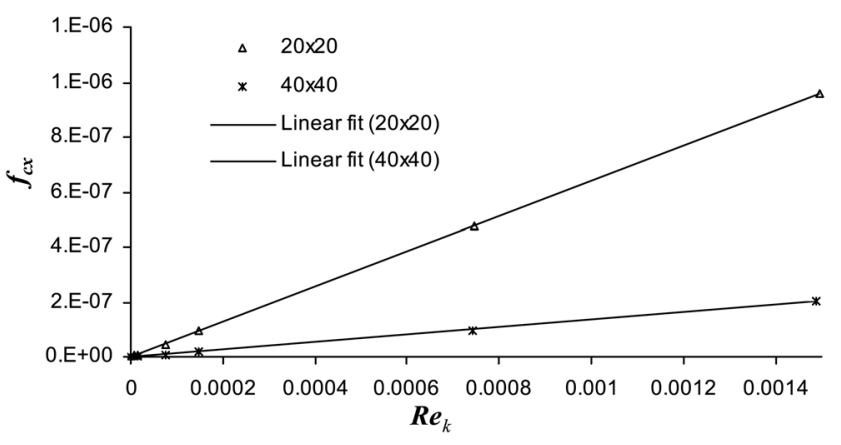

b)

FIG. 3. Variation of $f_{c x}=f_{x x}$ with $R e_{k}$, (a) for 4 different grid sizes. A power law fitted on points corresponding to $7.4 \times 10^{-4} \lesssim R e_{k} \lesssim 0.3$ gives an exponent 1.97 for $200 \times 200$ or $400 \times 400$ grid blocks; (b) the two coarser grids $20 \times 20$ and $40 \times 40$ reproduce an artificial linear variation of $f_{c x}$ with $R e_{k}$ at low Reynolds numbers. Unit cell of figure 1(b), $\varepsilon=0.75, \nabla\left\langle p_{\beta}^{*}\right\rangle^{\beta}=\mathbf{e}_{\mathbf{x}}$. 
and will not be further investigated (a few results were reported elsewhere ${ }^{39}$ ).

Although these fits provide excellent estimates in restricted ranges of the Reynolds number, it must be kept in mind, as discussed in the introduction, that this approach is based on approximations. This will be more clearly demonstrated with the dependence of the correction on the pressure gradient orientation as well as on disorder later investigated in this paper.

\section{Permeability and non-Darcy correction versus porosity: $\nabla\left\langle p_{\beta}^{*}\right\rangle^{\beta}=\mathbf{e}_{\mathbf{x}}$}

As a consequence of the accuracy test described above, all our computations on the model configuration of Figure 1(b) were performed with $150 \times 150$ grid blocks which is dense enough to analyze the values of the non-Darcy correction. The case $\nabla\left\langle p_{\beta}^{*}\right\rangle^{\beta}=\mathbf{e}_{\mathbf{x}}$ is first considered yielding a macroscale flow in the $x$ direction that is investigated for $0.3 \lesssim \varepsilon \leq 0.75$ and $R e_{k}$ up to around $14\left(R e_{d}\right.$ up to 70$)$.

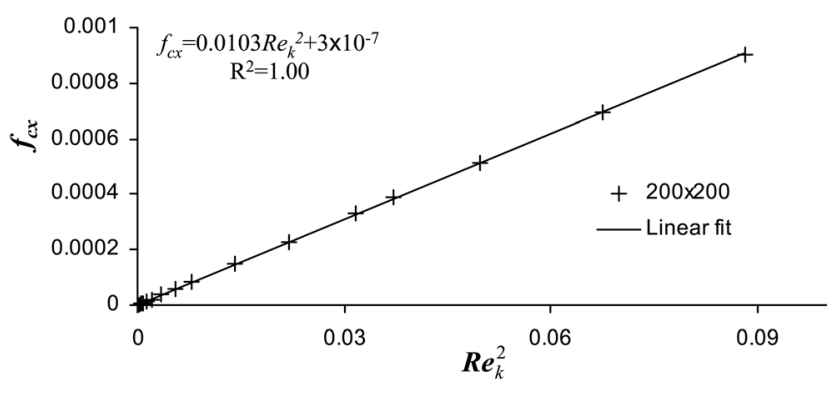

a)

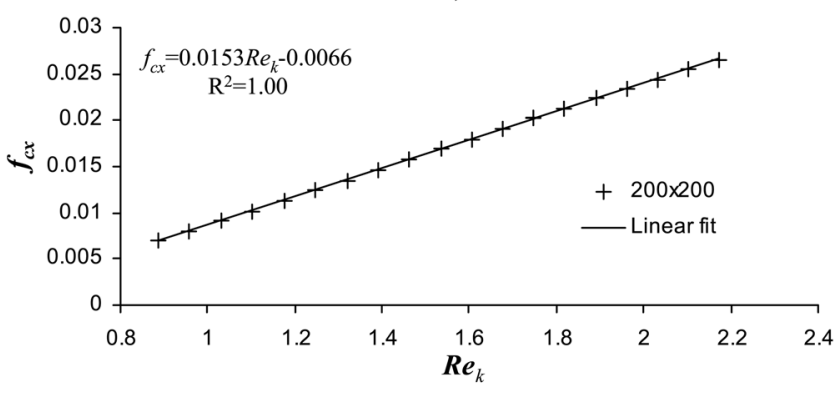

b)

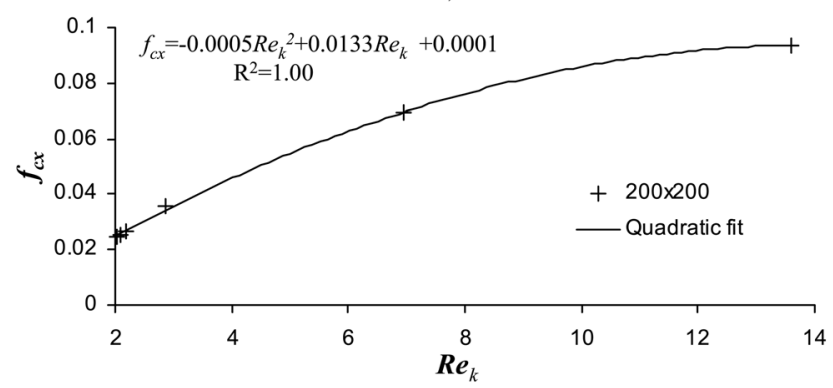

c)

FIG. 4. Correction to Darcy's law. (a) Weak inertia regime; $f_{c x}$ scales as $R e_{k}^{2}$ (i.e., $f_{i x}$ scales as $\left.\left(\left\langle\mathbf{v}_{\beta}\right\rangle . \mathbf{e}_{\mathbf{x}}\right)^{3}\right), 1.5 \times 10^{-4} \lesssim R e_{k} \lesssim 0.3$; (b) Strong inertia regime; $f_{c x}$ scales as $R e_{k}$ (i.e., $f_{i x}$ scales as $\left.\left(\left\langle\mathbf{v}_{\beta}\right\rangle \cdot \mathbf{e}_{\mathbf{x}}\right)^{2}\right), 0.9 \lesssim R e_{k} \lesssim 2.2$; (c) Regime above strong inertia, $f_{c x}$ scales as $R e_{k}^{2}, R e_{k} \gtrsim 2$. Unit cell of figure 1(b), $\varepsilon=0.75, \nabla\left\langle p_{\beta}^{*}\right\rangle^{\beta}=\mathbf{e}_{\mathbf{x}}$.
Results on $f_{c x}=f_{x x}$ versus $R e_{k}^{2}$ and $R e_{k}$ in the weak and strong inertia regimes are represented in Figure 5.

For the porosity range investigated here, these two regimes are well identified in restricted Reynolds number ranges. It must be pointed out that, for instance, for $\varepsilon=0.29$, $f_{c x}$ remains linear versus $R e_{k}$ only in the range $0.14 \lesssim R e_{k} \lesssim 0.33$. The distinction between the regimes was overlooked by Amaral Souto and Moyne ${ }^{50}$ in the same configuration with $\varepsilon=0.64$ because these authors analyzed the quantity $\varepsilon^{3} \frac{1}{\operatorname{Re}_{d} k^{*}}\left(1+f_{x x}\right)$. This quantity, proportional to the drag coefficient (or friction factor), is not sensitive enough to exhibit the expected non-linearity which remains small in this situation. As a consequence we further analyze the weak and strong inertia regimes by using the following linear representations for $f_{c x}$

$$
\begin{gathered}
f_{c x}=A \operatorname{Re}_{k}^{2}+B \quad(\text { weak inertia) }, \\
f_{c x}=C \operatorname{Re}_{k}+D \quad(\text { strong inertia }) .
\end{gathered}
$$

The corresponding values of the permeabilities $k^{*}$ along with the coefficients $A, B, C$ and $D$ are reported in Table II.

A short discussion on the $k^{*}-\varepsilon$ and $\beta-\varepsilon$ correlations is provided in Appendix B. As expected, for the whole range of porosity, $B$ is almost 0 clearly confirming a pure quadratic dependence of $f_{c x}$ upon $R e_{k}$ (i.e., a cubic dependence on velocity magnitude for $f_{i x}$ ) at the onset of the deviation from Darcy's law. In the strong inertia regime, where the correction $f_{i x}$ to Darcy's law has a quadratic dependence on
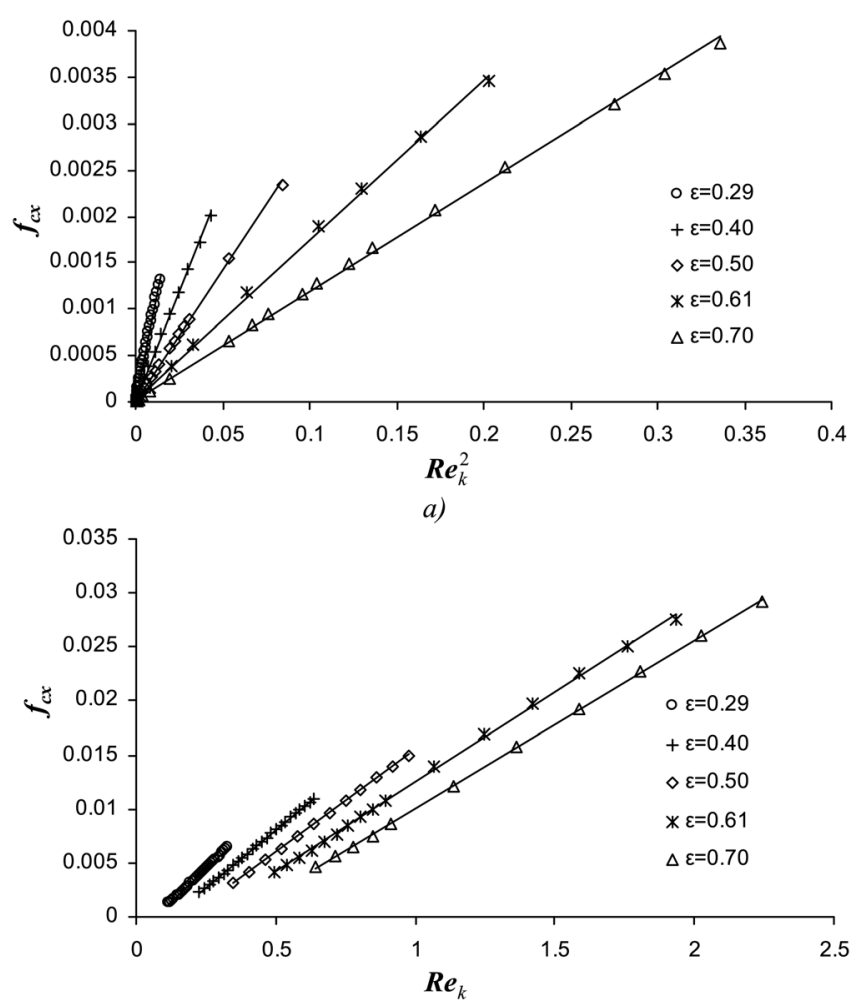

b)

FIG. 5. Variation of $f_{c x}$ with (a) $R e_{k}^{2}$ in the weak inertia regime, (b) $R e_{k}$ in the strong inertia regime, for $\varepsilon$ ranging from 0.29 to 0.75 . Unit cell of figure 1(b), $\nabla\left\langle p_{\beta}^{*}\right\rangle^{\beta}=\mathbf{e}_{\mathbf{x}}$. 
TABLE II. Permeabilities and coefficients in Eqs. (40) and (41) obtained from least square linear fits. Unit cell of Figure 1(b). Porosity range: $0.3 \lesssim \varepsilon \leq 0.75$.

\begin{tabular}{llcrcc}
\hline \hline$\varepsilon$ & \multicolumn{1}{c}{$\mathrm{k}^{*}$} & $\mathrm{~A}$ & \multicolumn{1}{c}{$\mathrm{B}$} & $\mathrm{C}$ & $\mathrm{D}$ \\
\hline 0.2944 & 0.0003651 & 0.1027 & $4 . \times 10^{-7}$ & 0.0251 & $-1.8 \times 10^{-3}$ \\
0.36 & 0.0007255 & 0.0649 & $2.0 \times 10^{-6}$ & 0.0228 & $-2.3 \times 10^{-3}$ \\
0.402 & 0.001068 & 0.0506 & $3 . \times 10^{-7}$ & 0.0215 & $-2.7 \times 10^{-3}$ \\
0.4425 & 0.001509 & 0.0397 & $7 . \times 10^{-7}$ & 0.0201 & $-2.9 \times 10^{-3}$ \\
0.5006 & 0.002386 & 0.0297 & $8 . \times 10^{-7}$ & 0.0190 & $-3.5 \times 10^{-3}$ \\
0.5466 & 0.003346 & 0.0241 & $1.0 \times 10^{-6}$ & 0.0180 & $-3.8 \times 10^{-3}$ \\
0.6073 & 0.005104 & 0.0187 & $3 . \times 10^{-7}$ & 0.0172 & $-4.6 \times 10^{-3}$ \\
0.6558 & 0.007059 & 0.0155 & $1 . \times 10^{-7}$ & 0.0165 & $-5.2 \times 10^{-3}$ \\
0.7012 & 0.009492 & 0.0125 & $3 . \times 10^{-7}$ & 0.0157 & $-5.7 \times 10^{-3}$ \\
0.75 & 0.013023 & 0.0103 & $1.3 \times 10^{-6}$ & 0.0153 & $-6.7 \times 10^{-3}$ \\
\hline \hline
\end{tabular}

velocity, a comparison between the inertial resistance factor $\beta$ in Eq. (3) and the coefficient $C$ obtained from our results can be easily performed. In fact, the classical Forchheimer model implicitly assumes no weak inertia regime and $D=0$ in Eq. (41). For this approximation to hold, a modified permeability-referred to as the Forchheimer permeabilitywhich differs from the true Darcy permeability, is generally introduced. ${ }^{44,48}$ Under such circumstances, the relation between $C$ and $\beta$ would be

$$
\frac{C}{\sqrt{k}}=\beta
$$

For a given $R e_{k}$, the intensity of inertia increases when $\varepsilon$ decreases. Moreover, the slopes of $f_{c x}\left(R e_{k}^{2}\right)$ and $f_{c x}\left(R e_{k}\right)$ decrease when $\varepsilon$ increases and this is much more significant in the weak inertia regime (see $A$ in Table II). Here it must be strongly emphasized that an analysis of this correction as a function of $R e_{d}^{2}$ and $R e_{d}$ would lead to exactly inverse conclusions on both intensity and slopes. The choice of the Reynolds number definition is therefore crucial. It must also be emphasized that, for this particular cell and pressure gradient configuration, the values of $f_{c x}$ in the weak inertia regime represent a quasi insignificant correction to Darcy's law.

A convenient way to describe the transition between the weak and strong inertia regimes is by defining a crossover Reynolds number $R e_{k c}$ as the value below and above which the best approximation is from Eqs. (40) and (41), respectively. This value is the one minimizing the difference between the weak and strong inertia models and is hence given by

$$
R e_{k c}=\frac{C}{2 A} .
$$

In order to analyze the range of $R e_{k}$ on which the relation (41) is a relevant approximation, an interval $\Delta R e_{k}$ is defined for all the situations investigated in the sequel of this work. It is delimited by $R e_{k c}$ and an upper bound for which numerical data are correlated by Eq. (41) within a 5\% error. Similarly, the transition region between weak and strong inertia is identified by $\delta R e_{k}$. The bounds of $\delta R e_{k}$ correspond to the values of $R e_{k}$ at which a 5\% deviation from the correlations (40) and (41) is obtained.

Results on $R e_{k c}$ and the corresponding values of $R e_{d c}$ that is simply given by

$$
R e_{d c}=\left(\frac{1-\varepsilon}{k^{*}}\right)^{0.5} \operatorname{Re}_{k c}
$$

are represented versus $\varepsilon$ in Figure 6(a). Clearly, these results show that the larger the porosity, the larger (respectively the smaller) the value of $R e_{k}$ (respectively $R e_{d}$ ) above which the correction, $f_{c x}$, depends linearly upon the Reynolds number. Moreover, as indicated in Figure 6(b), the range of Reynolds numbers $\Delta R e_{k}$ where this correction is a relevant approximation is increasing with $\varepsilon$ while the corresponding range $\Delta R e_{d}$ decreases. The correlations between each of the three quantities $R e_{k c}, R e_{d c}$ and $\Delta R e_{k}$ and $\varepsilon$ are presented in Appendix B.

In Figure 6(b), we have also reported the Reynolds number interval $\delta R e_{k}$ of the transition region between the weak and strong inertia regimes versus $\varepsilon$. Although 4 to 5 times smaller than $\Delta R e_{k}, \delta R e_{k}$ remains of significant extent.

In the next paragraph, pressure gradients not aligned with one of the periodicity axis of the unit cell are investigated for $\varepsilon=0.75$.

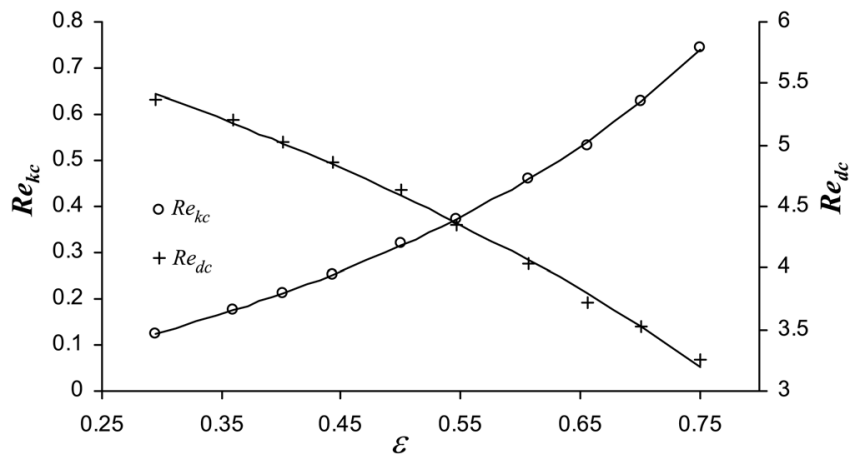

a)

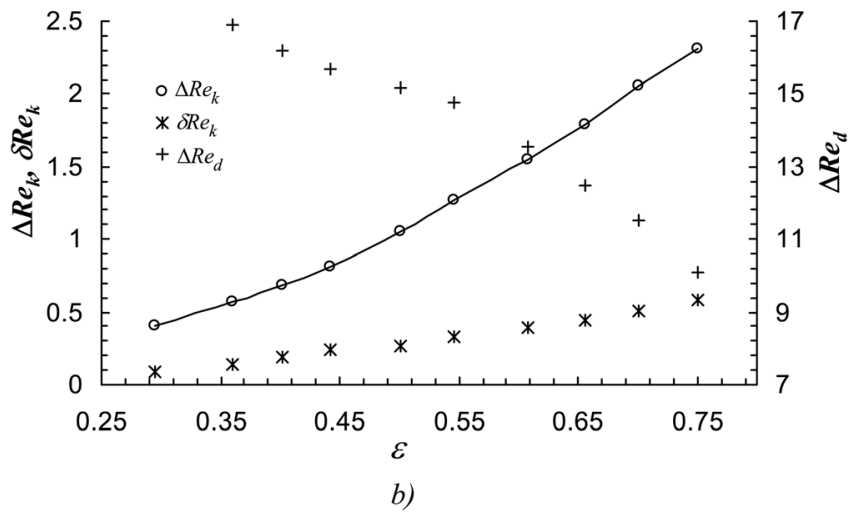

FIG. 6. (a) Variation of the crossover Reynolds number versus $\varepsilon$. Continuous lines are correlations of equations (B4) and (B5) provided in Appendix B. (b) Variation of the Reynolds number intervals $\Delta R e_{k}$ and $\Delta R e_{d}$ over which $f_{c x}$ varies linearly with $R e_{k}$ (or $R e_{d}$ ) versus $\varepsilon$. Continuous line corresponds to the correlation of equation (B6) given in Appendix B. $\delta R e_{k}$ is the Reynolds number interval of the transition between the weak and strong inertia regimes. Unit cell of figure $1(\mathrm{~b}) ; \nabla\left\langle p_{\beta}^{*}\right\rangle^{\beta}=\mathbf{e}_{\mathbf{x}}$. 


\section{Non-Darcy correction versus $\nabla\left\langle\boldsymbol{p}_{\beta}^{*}\right\rangle^{\beta}$ orientation:
$\varepsilon=0.75$}

When $\nabla\left\langle p_{\beta}^{*}\right\rangle^{\beta}$ is not aligned with $\mathbf{e}_{\mathbf{x}}$ or $\mathbf{e}_{\mathbf{y}}$, the computation of the tensor $\mathbf{F}$ requires the use of the full algorithm described in Sec. II B. This was performed for $\varepsilon=0.75$ in the range $0 \leq R e_{d} \leq 30\left(R e_{k}\right.$ up to 10$)$ and $0 \leq \theta \leq 45^{\circ}$, where $\theta$ designates the angle $\nabla\left\langle p_{\beta}^{*}\right\rangle^{\beta}$ makes with $\mathbf{e}_{\mathbf{x}}$. Results in the range $45^{\circ} \leq \theta \leq 90^{\circ}$ can be inferred from evident symmetry considerations according to $f_{x x}\left(90^{\circ}-\theta\right)=f_{y y}(\theta)$, $f_{x y}\left(90^{\circ}-\theta\right)=f_{y x}(\theta)$ and similarly $f_{c y}(\theta)=f_{c x}\left(90^{\circ}-\theta\right)$, $\left|\mathbf{f}_{\mathbf{c}}(\theta)\right|=\left|\mathbf{f}_{\mathbf{c}}\left(90^{\circ}-\theta\right)\right|, 0^{\circ} \leq \theta \leq 90^{\circ}$.

a. $F$ tensor analysis. The components of $\mathbf{F}$ versus $\theta$ and $R e_{k}$ are represented in Figure 7(a). As expected, $\mathbf{F}$ is not symmetric (see Figure 7(b) representing $f_{x y}-f_{y x}$ versus $\theta$ and $R e_{k}$ ) except for $\theta=0^{\circ}, \theta=90^{\circ}$ and $\theta=45^{\circ}$ all the three corresponding to a symmetry axis while, additionally, $f_{x x}=f_{y y}$ for the latter value. This last feature can be stated in the following general manner. When, for an isotropic structure, the pressure gradient is along a symmetry axis of the cell and leads to a flow for which the principal axes of $\mathbf{H}$ and $\mathbf{F}$ are not the periodicity axes $\mathbf{e}_{\mathbf{x}}$ and $\mathbf{e}_{\mathbf{y}}$, then $\mathbf{H}$ and $\mathbf{F}$ are symmetric with $h_{x x}=h_{y y}$ and $f_{x x}=f_{y y}$. The nonsymmetric character of $\mathbf{F}$ increases with $R e_{k}$. At very low Reynolds numbers, the $f_{x y}-f_{y x}$ extrema are obtained for the two remarkable values $22.5^{\circ}$ and $67.5^{\circ}$ of $\theta$.

These results suggest that, except when the flow is along one of the symmetry axes, the mean velocity $\left\langle\mathbf{v}_{\beta}^{*}\right\rangle$ is not in the direction of $\nabla\left\langle p_{\beta}^{*}\right\rangle^{\beta}$, i.e., that the macroscopic force exerted on the structure is not pure drag. This is confirmed by the results on the angle $\left\langle\mathbf{v}_{\beta}^{*}\right\rangle$ makes with $\nabla\left\langle p_{\beta}^{*}\right\rangle^{\beta}$ discussed below. It must be noted that this property might be inherent to the ordered structure of the medium as mentioned elsewhere. ${ }^{64}$

b. Mean flow versus $\nabla\left\langle p_{\beta}^{*}\right\rangle^{\beta}$ orientation. In Figure 7(c), we have represented the variation of $\theta_{v}$ versus $\theta$ and $R e_{k}, \theta_{v}$ being the angle $\left\langle\mathbf{v}_{\beta}^{*}\right\rangle$ makes with $\nabla\left\langle p_{\beta}^{*}\right\rangle^{\beta}$. This angle features complex dependence on $\theta$ and $\operatorname{Re}_{k}$. From Figure 7(c) it can be observed that, globally, for $0<\theta<45^{\circ}$, the flow tends to be more aligned with the $x$ direction than $\nabla\left\langle p_{\beta}^{*}\right\rangle^{\beta}$ $\left(\theta_{v}\right.$ is positive), whereas for $45^{\circ}<\theta<90^{\circ},\left\langle\mathbf{v}_{\beta}^{*}\right\rangle$ tends to
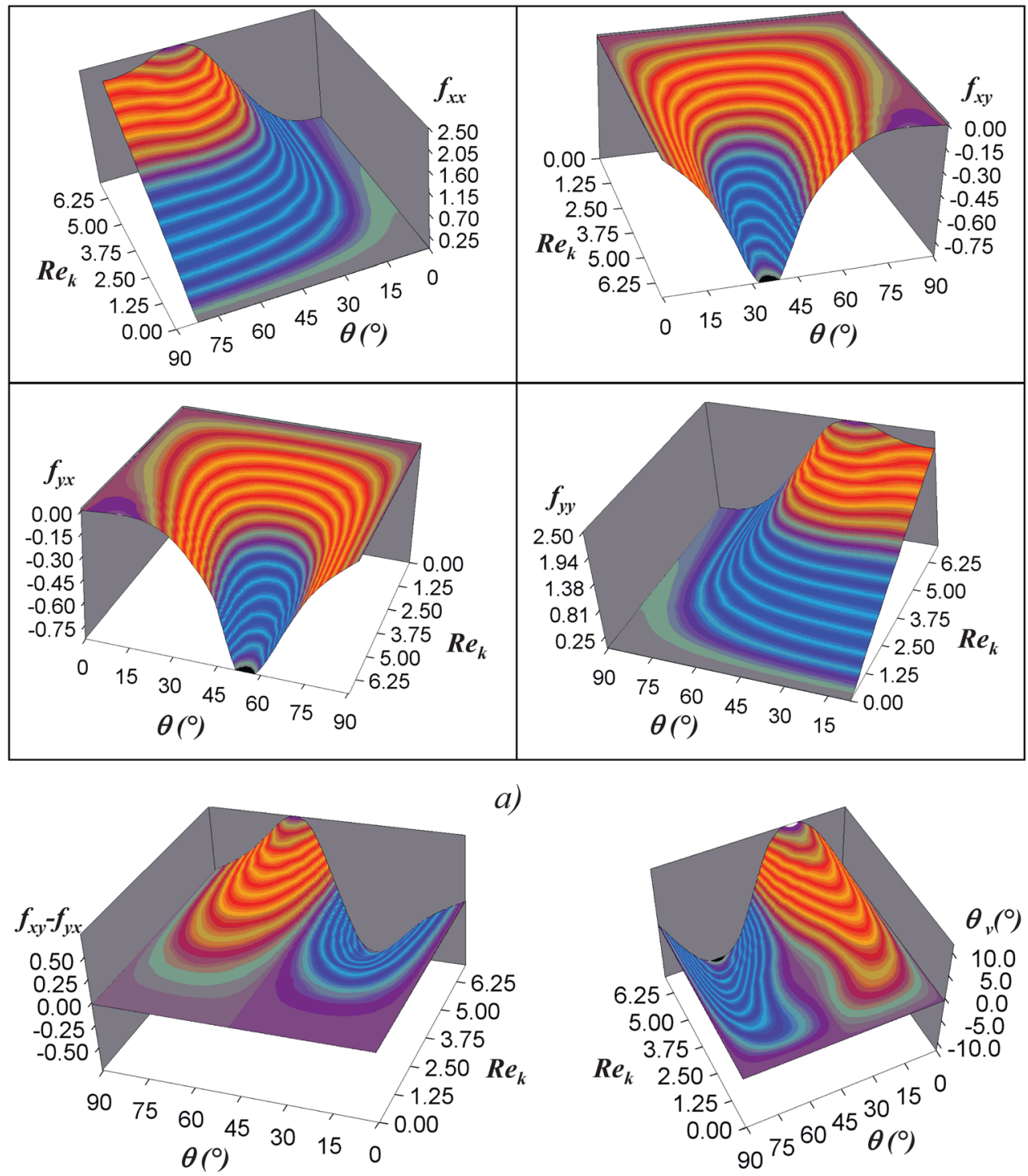

b)

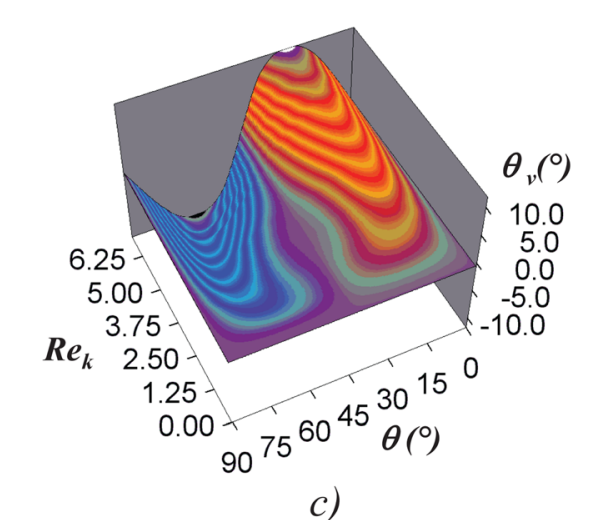

c)

a)
FIG. 7. (Color online) Properties of the $\mathbf{F}$ tensor and mean flow versus $\theta$ and $R e_{k}$. (a) Components of F. (b) Difference between the off-diagonal terms of F. (c) Angle $\theta_{v}$ between $\left\langle\mathbf{v}_{\beta}^{*}\right\rangle$ and $\nabla\left\langle p_{\beta}^{*}\right\rangle^{\beta}$. Pure drag only exists for $\theta=0^{\circ}, 45^{\circ}$, and $90^{\circ} . \theta_{v}(\theta)=-\theta_{v}(90-\theta)$ for $0^{\circ} \leq \theta \leq 90^{\circ}$. Unit cell of figure $1(\mathrm{~b}) ; \varepsilon=0.75$. 
align with the $y$ direction. The maximum value of $\theta_{v}$ always increases with $R e_{k}$. The value of $\theta$ for which $\theta_{v}$ is maximum is equal to $22.5^{\circ}$ at small $R e_{k}$, decreases slightly for $R e_{k}$ up to $\sim 3$ and increases again for larger $R e_{k}$. We shall now examine the consequences on the nonDarcy correction.

c. Non-Darcy correction versus $\nabla\left\langle p_{\beta}^{*}\right\rangle^{\beta}$ orientation. Because of symmetry, our conclusions will only be drawn on $f_{c x}$ and $\left|\mathbf{f}_{\mathbf{c}}\right|$ in the rest of this paragraph. In Figures 8(a) and $8(\mathrm{~b})$, we have reported the variation of these two quantities, respectively, versus $\theta$ and $R e_{k}$. Due to symmetry, the maximum of $\left|\mathbf{f}_{\mathbf{c}}\right|$ is for $\theta=45^{\circ}$ whatever the value of $R e_{k}$. However, this is not the case for $f_{c x}$ since the orientation of $\nabla\left\langle p_{\beta}^{*}\right\rangle^{\beta}$ leading to the maximum value of this component depends on the Reynolds number. For instance, the value of $\theta$ corresponding to the maximum of $f_{c x}$ decreases (for $R e_{k}$ up to roughly 3.3, see inset of Figure $8(\mathrm{a}))$ and increases (asymptotically) to $\theta=45^{\circ}$ at large $R e_{k}$. The correction is always minimum for $\theta=0^{\circ}$ (or $\theta=90^{\circ}$ ) whatever the Reynolds number.

Clearly, $f_{c x}$ scales as $R e_{k}^{2}$ for small enough values of $R e_{k}$ at any orientation $\theta$ of $\nabla\left\langle p_{\beta}^{*}\right\rangle^{\beta}$ as shown in Figure 9 (a similar behaviour is obtained for $\left.\left|\mathbf{f}_{\mathbf{c}}\right|\right)$. The excellent linear dependence confirms the existence of the weak inertia regime.

However, inspecting the behavior of $f_{c x}$ and $\left|\mathbf{f}_{\mathbf{c}}\right|$ for larger Reynolds numbers indicates that the existence of a linear dependence on $R e_{k}$ in a strong inertia regime, when it can be identified, is only an approximation. This is obvious

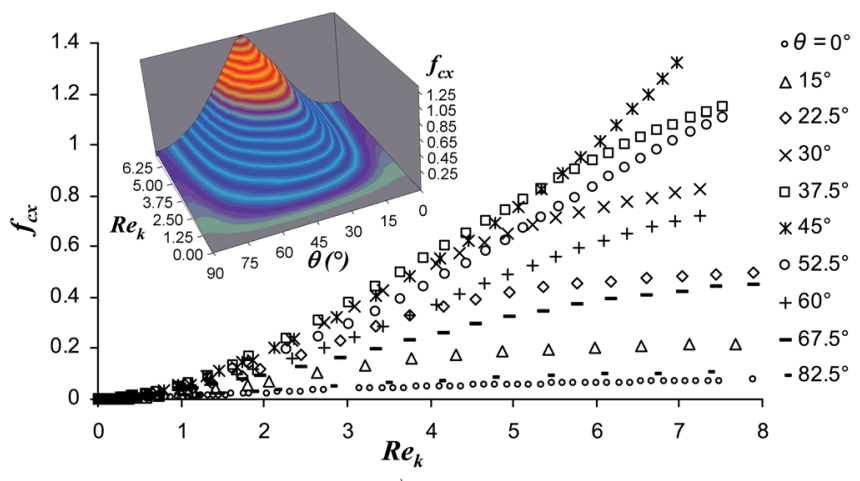

a)

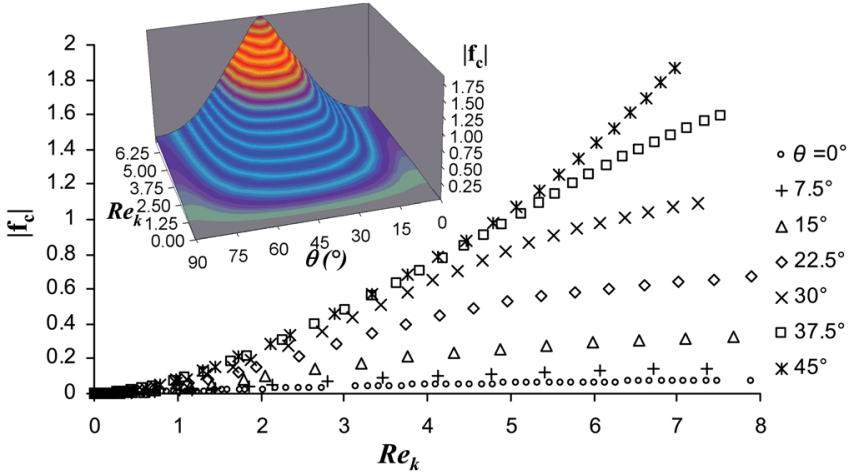

b)

FIG. 8. (Color online) Variation of (a) the $x$ component, $f_{c x}$, of $\mathbf{f}_{\mathbf{c}}$ and (b) the modulus of $\mathbf{f}_{\mathbf{c}}$ with $R e_{k}$ for different values of $\theta$. Insets: same quantities versus $\theta$ and $R e_{k}$. Unit cell of figure $1(\mathrm{~b}) ; \varepsilon=0.75$.

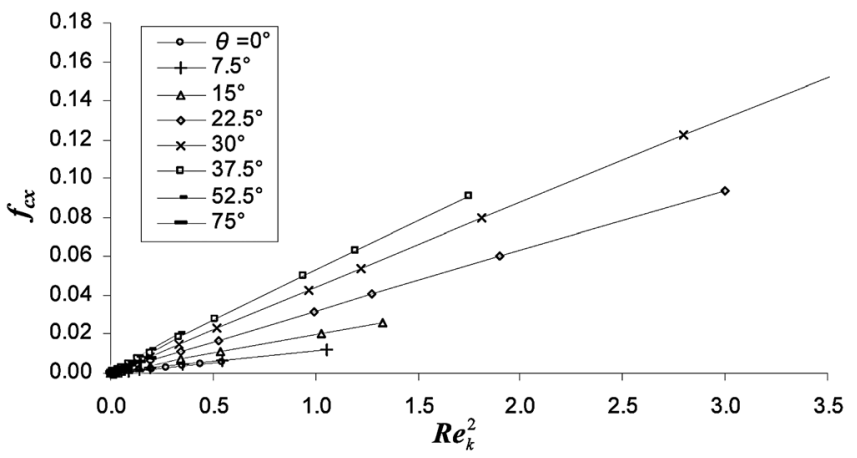

FIG. 9. Variation of the $x$ component, $f_{c x}$, of $\mathbf{f}_{\mathbf{c}}$ with $R e_{k}^{2}$ for different values of $\theta$. Unit cell of figure $1(\mathrm{~b}) ; \varepsilon=0.75$.

when the derivatives of these quantities are represented versus $R e_{k}$ as reported in Figures 10(a) and 10(b) respectively. Evidently, the relationship between $f_{c x}$ (or $\left|\mathbf{f}_{\mathbf{c}}\right|$ ) and $R e_{k}$ is reasonably linear over a restricted Reynolds number interval centered on the inflexion point of this relationship.

The case $\theta=45^{\circ}$ is a singular situation for which no inflexion point is obtained for $R e_{k}$ up to $\sim 7\left(R e_{d} \sim 30\right)$ whereas a quadratic law fits very well the data in the whole range of Reynolds numbers investigated here as

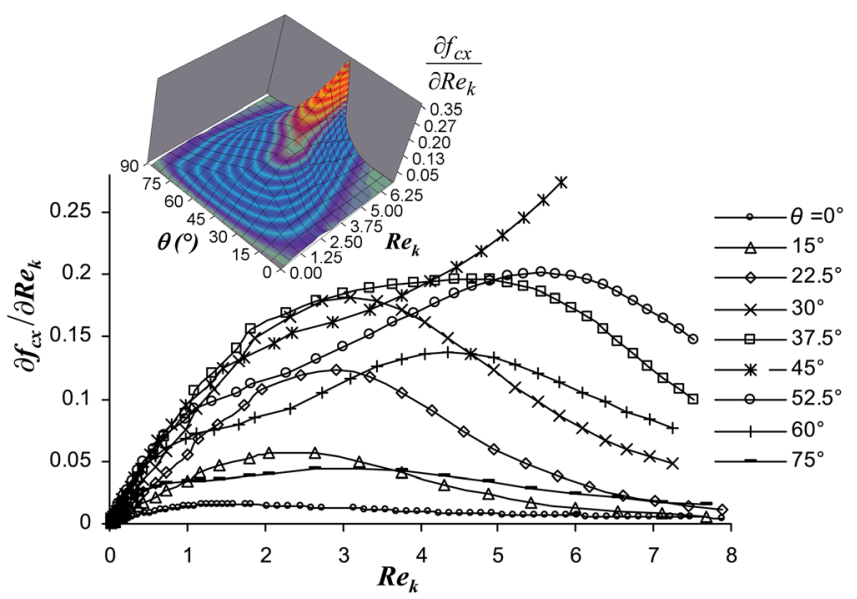

a)

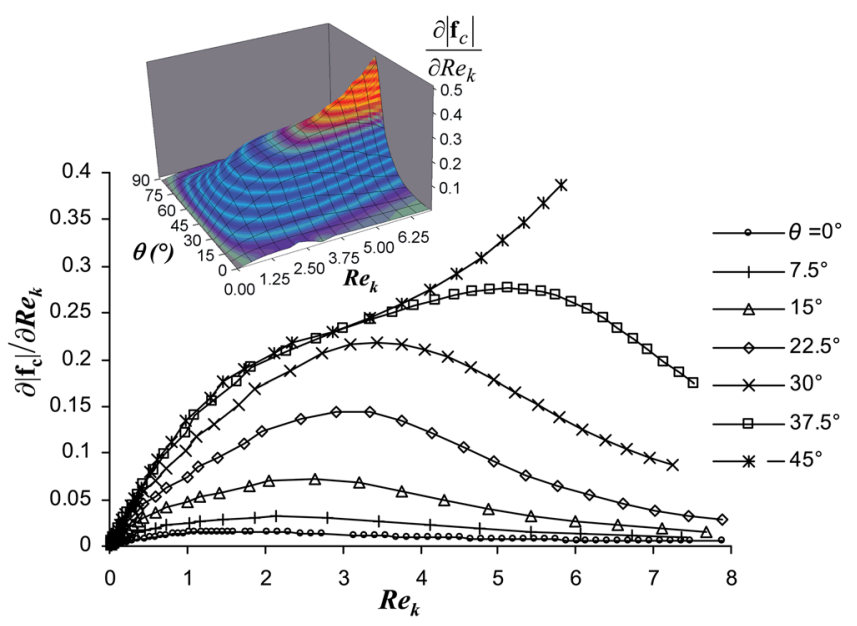

b)

FIG. 10. (Color online) Variation of (a) $\frac{\partial f_{c x}}{\partial e_{k}}$ and (b) $\frac{\partial\left|\mathbf{f}_{\mathrm{c}}\right|}{\partial R e_{k}}$ with $R e_{k}$ for different values of $\theta$. Insets: same quantities versus $\theta$ and $R e_{k}$. Unit cell of Figure $1(\mathrm{~b}) ; \varepsilon=0.75$. 
demonstrated in Figure 11. The existence of a linear dependence of the correction on $R e_{k}$ for higher values of the Reynolds number, although plausible, is however questionable regarding the non-stationary character of the flow that may occur in a higher velocity range.

For both $f_{c x}$ and $\left|\mathbf{f}_{\mathbf{c}}\right|$, the inflexion zone shifts to higher Reynolds numbers (see Figure 10(a) and 10(b) when $\theta$ increases from $0^{\circ}$ to values close to $45^{\circ}$. On the contrary, when $\theta$ increases from $45^{\circ}$ to $90^{\circ}$, the inflexion zone shifts to lower Reynolds numbers.

Identification of weak and strong inertia regimes for both $f_{c x}$ and $\left|\mathbf{f}_{\mathbf{c}}\right|$ and all angles (except for strong inertia at $\theta=45^{\circ}$ ) allows the determination of the coefficients $A, B, C$ and $D$ in correlations (40) and (41). These coefficients are represented in Figures 12(a) and 12(b). As expected, they are symmetric about $\theta=45^{\circ}$ when estimated on $\left|\mathbf{f}_{\mathbf{c}}\right|$; they are not when estimated on $f_{c x}$. Results in the weak inertia regime indicate that

(i) $\quad B$ is always extremely small (less than $10^{-5}$ ) confirming that the onset of deviation from Darcy's law scales as $R e_{k}^{2}$ for $\mathbf{f}_{\mathbf{c}}$ (i.e., as $\left|\left\langle\mathbf{v}_{\beta}\right\rangle\right|^{3}$ for $\mathbf{f}_{\mathbf{i}}$ ).

(ii) Intensity of inertial effects strongly depends on $\theta$ since $A$ varies over one order of magnitude for both $f_{c x}$ and $\left|\mathbf{f}_{\mathbf{c}}\right|$. The correction can be significant since the drag due to weak inertia can be up to $20 \%$ of the drag in the Darcy regime.

In the strong inertia regime, it can be concluded that

(i) $D$ in the correlation (41) is not negligible, in particular when $\theta$ approaches $45^{\circ}$. This confirms that the Forchheimer type of correction is an approximation for this ordered structure (see the above discussion while introducing the relationship (42)). In fact the use of a pure quadratic velocity correction for $\mathbf{f}_{\mathbf{i}}$ requires the contribution of $D$ to be lumped in a Forchheimer permeability different from the intrinsic one.

(ii) Inertial effects strongly increase with $\theta$ up to $45^{\circ}$ and then decrease. The ratio of the maximum to minimum values of $C$ for $f_{c x}$ and $\left|\mathbf{f}_{\mathbf{c}}\right|$ is about 10 and 20, respectively (see Figure 12(a)).

Crossover Reynolds numbers $R e_{k c}$ determined from $A$ and $C$ (see Eq. (43)) are represented in Figure 13(a). Clearly,

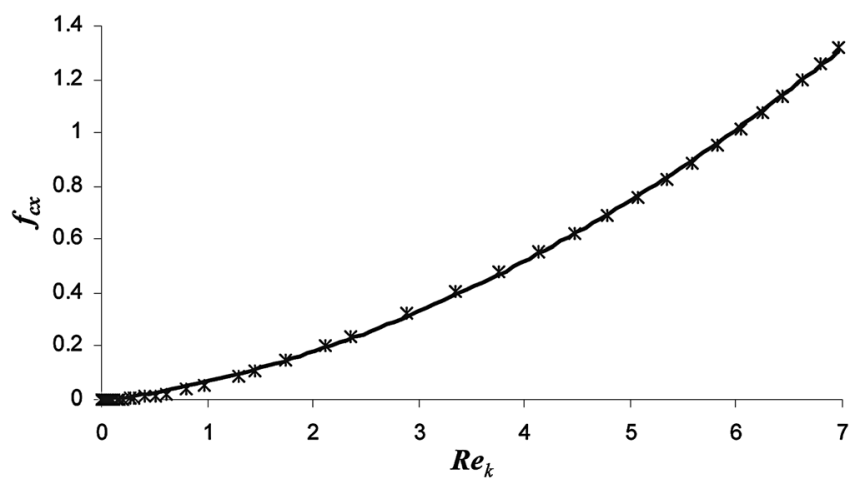

FIG. 11. Variation of $f_{c x}$ as a function of $R e_{k}$ for $\theta=45^{\circ}$ and $0 \leq R e_{k} \leq 7$. Unit cell of figure $1(\mathrm{~b}) ; \varepsilon=0.75$. Symbols: numerical results. Continuous line: quadratic correlation $f_{c x}=0.0192 R e_{k}^{2}+0.0545 R e_{k}-0.0056 ; R^{2}=$ 0.9997 .
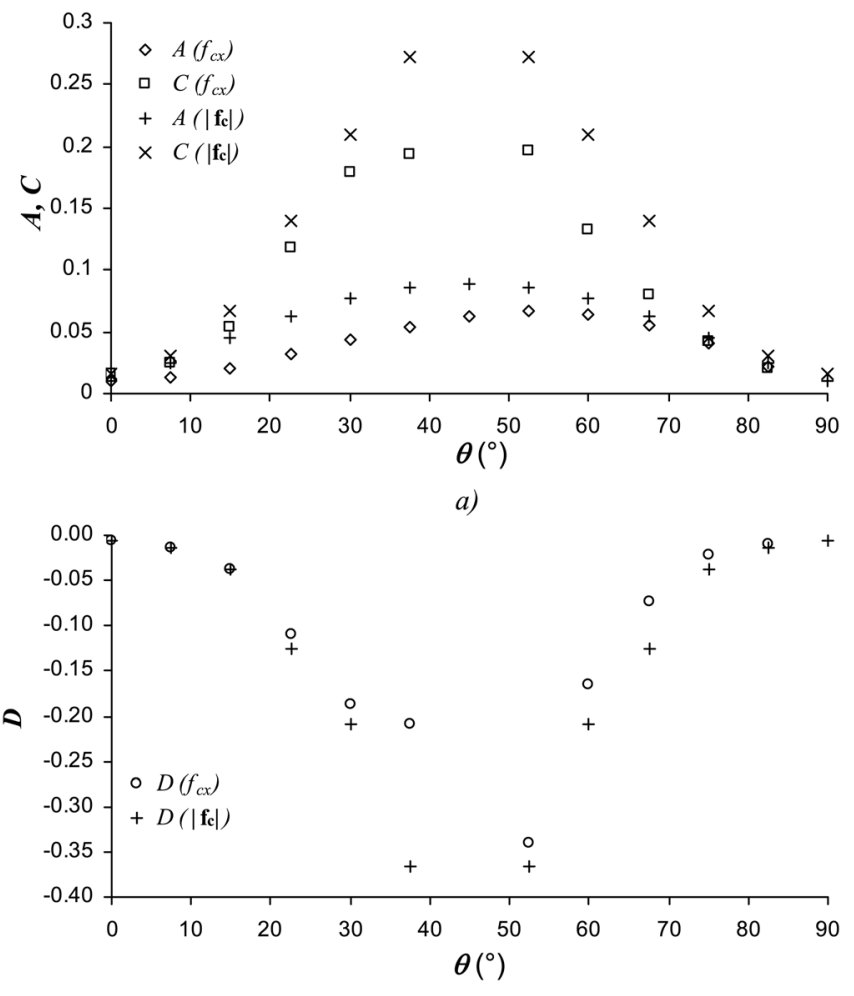

b)

FIG. 12. Coefficients (a) $A, C$ and (b) $D$ of correlations (40) and (41) versus $\nabla\left\langle p_{\beta}^{*}\right\rangle^{\beta}$ orientation $\theta$. Unit cell of Figure $1(\mathrm{~b}) ; \varepsilon=0.75$.

the pressure gradient orientation influences very significantly $R e_{k c}$ that is larger when $\theta$ is close to $45^{\circ}$ (respectively $30^{\circ}$ ) considering $\left|\mathbf{f}_{\mathbf{c}}\right|$ (respectively $f_{c x}$ ). The variation of $\Delta R e_{k}$ with $\theta$ is represented in Figure 13(b) where we have also reported the intervals $\delta R e_{k}$ corresponding to the transition between this regime and weak inertia.

Both $\Delta R e_{k}$ and $\delta R e_{k}$ increase for $\theta$ up to $45^{\circ}$ and then decrease but are not defined for $\theta=45^{\circ}$ in accordance with the result in Figure 11. Emphasis must be put on the ratio of $\Delta R e_{k}$ to $\delta R e_{k}$ which can vary from $\sim 2$ to $\sim 4$ for different values of $\theta$ considering either $f_{c x}$ or $\left|\mathbf{f}_{\mathbf{c}}\right|$. This indicates that the transition zone between weak and strong inertia regimes is generally not negligible.

A further study on two-dimensional disordered structures is carried out in the next section.

\section{B. Structural disorder}

In this section, results on $\mathbf{f}_{\mathbf{c}}$ when disorder is introduced both on position and size of solid inclusions are compared to those in the ordered case for a given porosity $(\varepsilon=0.75)$. The case $\theta=0^{\circ}$, i.e., $\nabla\left\langle p_{\beta}^{*}\right\rangle^{\beta}=\mathbf{e}_{\mathbf{x}}$, is only considered.

\section{Structure generation}

Weakly and strongly disordered structures were generated on the basis of the ordered unit cell used so far.

a. Weak Disorder (WD). Weak structural disorder is understood here as the result of random placement of the center of the square solid inclusion in the cell of Figure 1(b) 


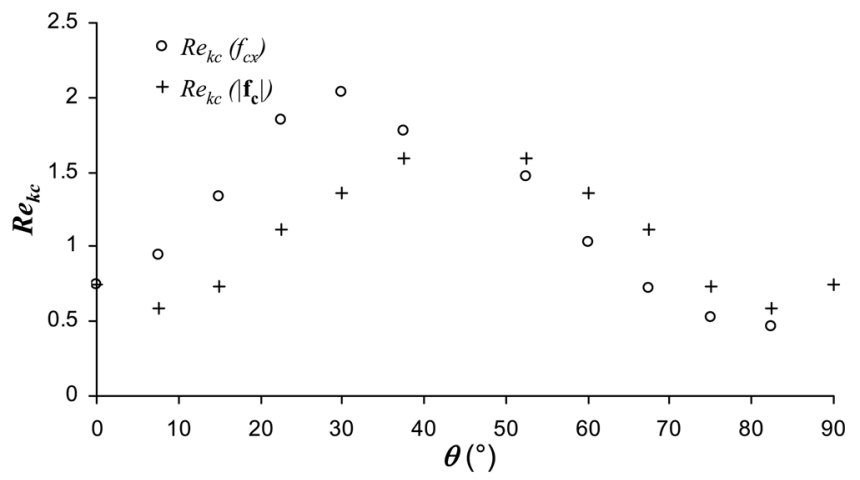

a)

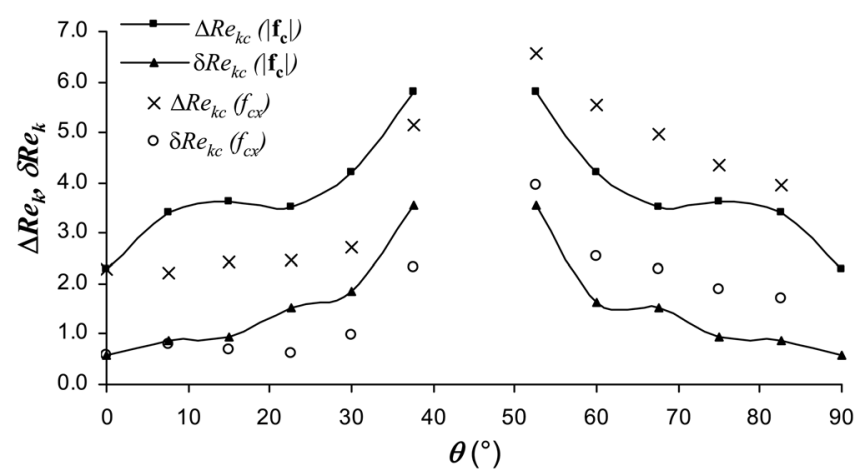

b)

FIG. 13. Dependence upon $\nabla\left\langle p_{\beta}^{*}\right\rangle^{\beta}$ orientation, $\theta$, of (a) the crossover Reynolds number $R e_{k c}$ (it is not defined for $\theta=45^{\circ}$ ), $b$ ) the Reynolds number interval $\Delta R e_{k}$ over which $f_{c x}$ and $\left|\mathbf{f}_{\mathbf{c}}\right|$ variations with $R e_{k}$ are linearly approximated. $\delta R e_{k}$ is the Reynolds number interval of the transition between the weak and strong inertia regimes. Unit cell of figure $1(b) ; \varepsilon=0.75$.

according to a uniform distribution. All dimensions $(l$ and d) were kept the same. To avoid extremely narrow channels requiring a large number of grid blocks, random placement was constrained within a centered sub-domain of size $0.85 l$ in each cell. The unit cell of the periodic weakly disordered porous structure was obtained by reassembling $n \times n$ random cells of edge size $l$ resulting from this process. An example with $n=10$ is depicted in Figure 14(a).

b. Strong Disorder ( $S D)$. Strong disorder was obtained by a random choice of the size of the solid square inclusions superimposed to their random placement in the cell of size $l$ as described above. The same random generator was used for both random processes and the porosity was constrained to the fixed value of 0.75 . The inclusion edge size was chosen in the range $[0.125 l, 0.75 l]$ again to avoid narrow fluid channels. The final periodic unit cell was obtained as before by reassembling $n \times n$ random cells. A realization with $n=10$ is represented in Figure 14(b).

When representative, $W D$ and $S D$ unit cells should reproduce the expected isotropic character of the infinite porous structure having the same random properties as the realization since the random generator is direction independent. To meet this feature, ergodicity hypothesis can be invoked so that the expected values of $\mathbf{K}^{*}$ and $\mathbf{F}$ are those obtained by averaging the corresponding quantities obtained on a large enough number of unit cells smaller than a repre-

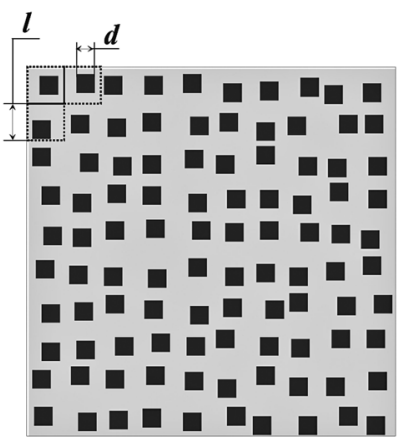

a)

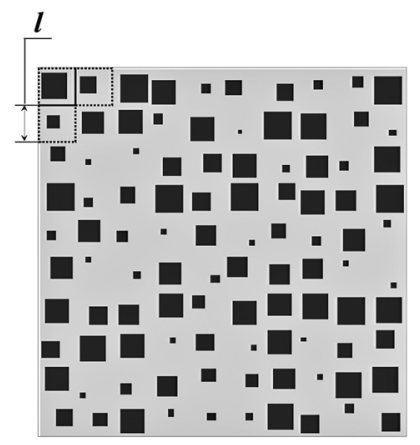

b)
FIG. 14. Unit cell containing $10 \times 10$ square solid inclusions. $\varepsilon=0.75$. (a) Weakly disordered $(W D)$ structure with random placement of solid inclusions. (b) Strongly disordered (SD) structure with random placement of solid inclusions of random size.

sentative one. Hence, $\mathbf{K}^{*}$ and $\mathbf{F}$ were computed over ten different realizations for both $W D$ and $S D$ structures. Unit cells with $n=10$ and $400 \times 400$ grid blocks were used, allowing a good compromise between computational resources and accuracy requirements. Tests were also performed on $W D$ unit cells with $n=10$ and $800 \times 800$ grid blocks to check accuracy as well as $n=20$ and $800 \times 800$ grid blocks to check ergodicity. Accuracy criteria were those afore mentioned. Simulations were carried out for $R e_{k}$ up to $\sim 7$ (i.e. $R e_{d}$ up to $\sim 31)$. For each SD realization, $R e_{d}$ was calculated from the average value of $d$. An example of a velocity magnitude contour map obtained on a WD structure is depicted in Figure 15 which clearly highlights channeling through less resistance flow paths resulting from disorder.

\section{Permeability}

Average values of the diagonal $\left(\overline{k^{*}}=0.5\left(\overline{k_{x x}^{*}}+\overline{k_{y y}^{*}}\right)\right)$ and off diagonal $\left(\overline{k_{x y}^{*}}\right)$ terms computed on the ten realizations of each structure as well as corresponding standard

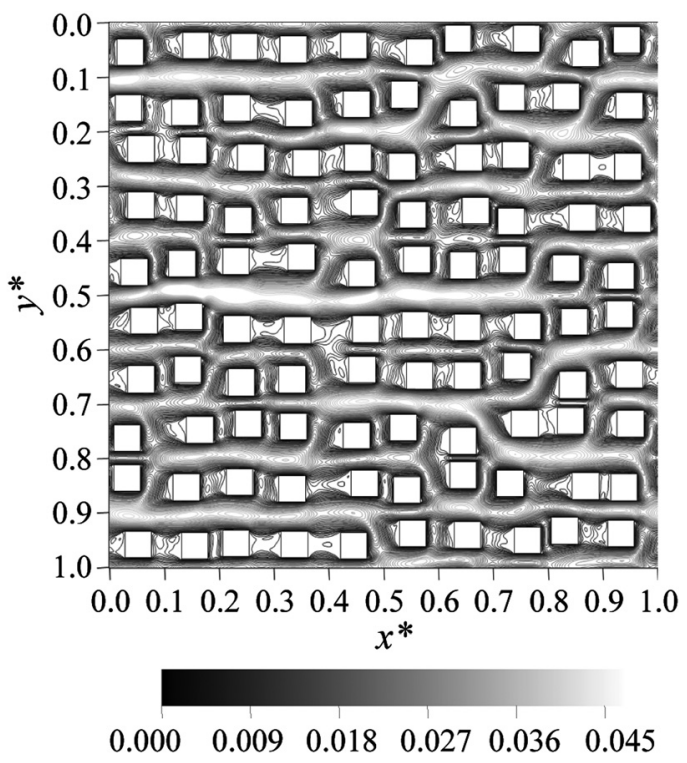

FIG. 15. Velocity magnitude contour map obtained on unit cell of Figure 14(a). $\nabla\left\langle p_{\beta}^{*}\right\rangle^{\beta}=\mathbf{e}_{\mathbf{x}}, \operatorname{Re}_{k}=2.48,\left(\operatorname{Re}_{d}=10.80\right)$. 
TABLE III. Average values of the components of $\mathbf{K}^{*}$ obtained on ten different unit cell realizations of $W D$ and $S D$ structures (see Figures 14(a) and 14(b)). $\varepsilon=0.75, \nabla\left\langle p_{\beta}^{*}\right\rangle \beta=\mathbf{e}_{\mathbf{x}}, n=10,400 \times 400$ grid blocks.

\begin{tabular}{lcc}
\hline \hline Structure & $\overline{k^{*}}(\sigma)$ & $\overline{k_{x y}^{*}}(\sigma)$ \\
\hline WD & $0.01308\left(3.82 \times 10^{-4}\right)$ & $-1.01 \times 10^{-4}\left(4.75 \times 10^{-4}\right)$ \\
SD & $0.01350\left(7.72 \times 10^{-4}\right)$ & $-3.43 \times 10^{-4}\left(6.69 \times 10^{-4}\right)$ \\
Ordered & 0.01304 & - \\
\hline \hline
\end{tabular}

deviations, $\sigma$, are gathered in Table III. For both $W D$ and $S D$ cases, $\overline{k^{*}}$ is very close to the value obtained on the ordered structure for the same grid size with a slight increase while increasing disorder. In comparison, the standard deviations are very small showing the narrow distribution of $k_{x x}^{*}$ and $k_{y y}^{*}$. The off-diagonal term $k_{x y}^{*}$ has almost the same standard deviation but a mean value $\overline{k_{x y}^{*}}$ roughly two orders of magnitude smaller than $\overline{k^{*}}$ indicating that the set of realizations reasonably restores isotropy. The accuracy test performed on the same $W D$ unit cells with $n=10$ and $400 \times 400$ grid blocks on the one hand and $800 \times 800$ grid blocks on the other hand leads to a relative error of $\sim 6 \times 10^{-2} \%$ and $\sim 7 \times 10^{-2} \%$ on the diagonal and off-diagonal terms respectively. Moreover, the permeability tensor computed on $W D$ unit cells with $n=20$ and $800 \times 800$ grid blocks yields average values of $1.319 \times 10^{-2}$ on the diagonal terms (i.e., a relative error of $0.8 \%)$ and $7.83 \times 10^{-5}$ on the off-diagonal terms. This confirms the expected isotropic character of the cell in the Darcy

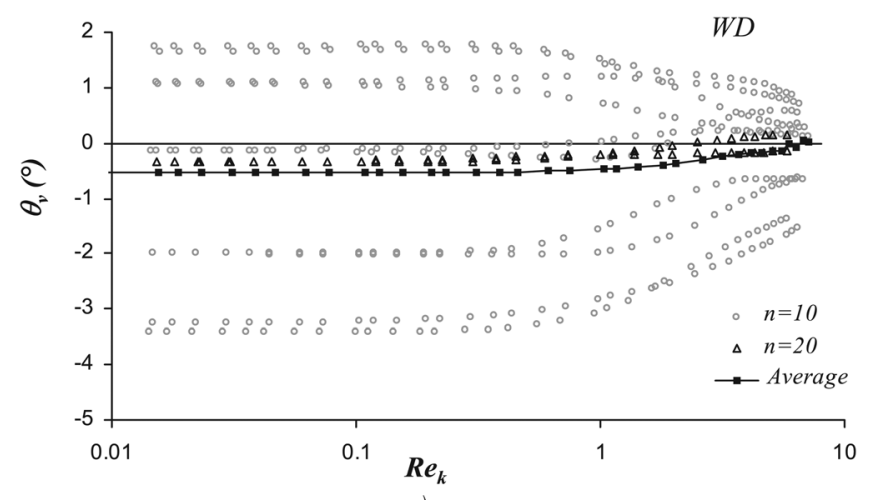

a)

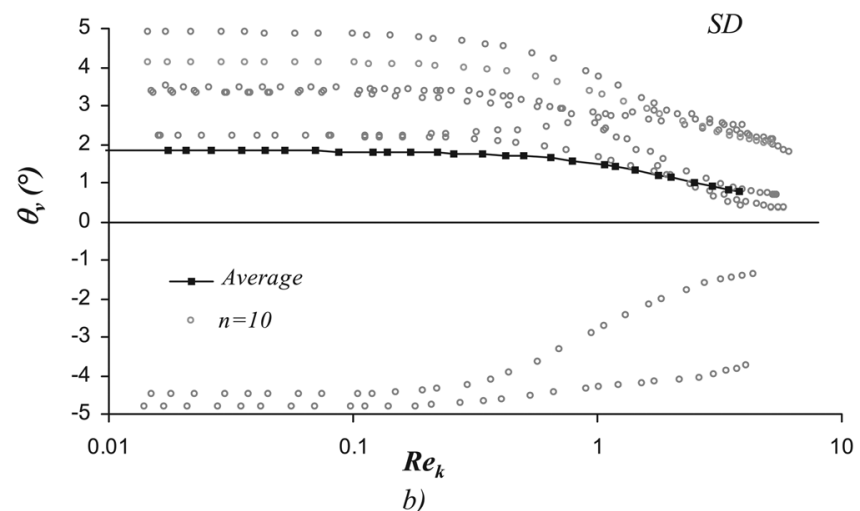

FIG. 16. Angle $\theta_{v}$ between $\left\langle\mathbf{v}_{\beta}^{*}\right\rangle$ and $\nabla\left\langle p_{\beta}^{*}\right\rangle^{\beta}$ and average value of $\theta_{v}$ versus $R e_{k}$ for ten different realizations with $n=10$ of (a) the $W D$ unit cell, results for two realizations with $20 \times 20$ inclusions are also reported and (b) the $S D$ unit cell. $\varepsilon=0.75, \nabla\left\langle p_{\beta}^{*}\right\rangle^{\beta}=\mathbf{e}_{\mathbf{x}}$.

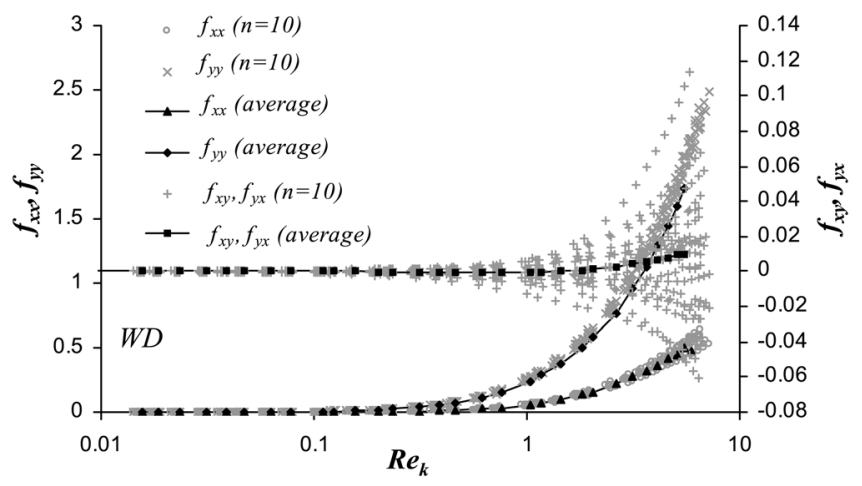

a)

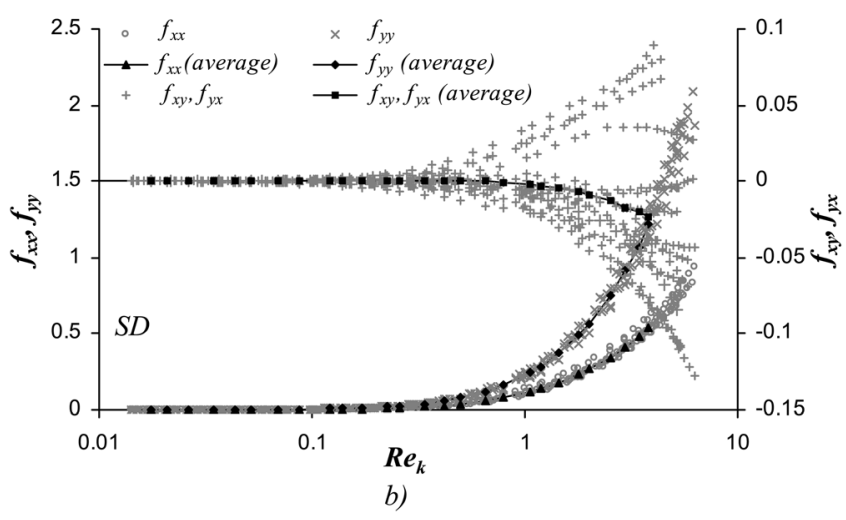

FIG. 17. Variation of the components of $\mathbf{F}$ with $R e_{k}$ for ten different realizations of (a) the $W D$ unit cell and (b) the $S D$ unit cell. $\varepsilon=0.75, \nabla\left\langle p_{\beta}^{*}\right\rangle^{\beta}=\mathbf{e}_{\mathbf{x}}$.
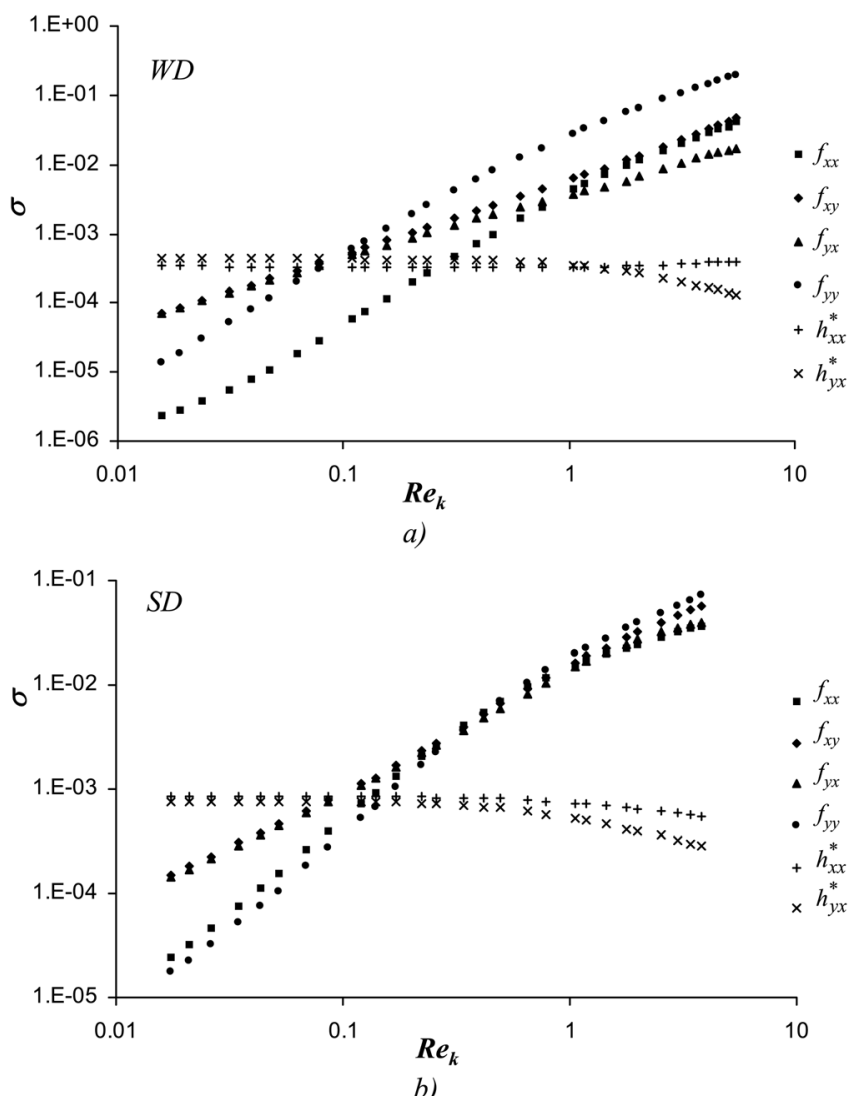

FIG. 18. Variation with $R e_{k}$ of the standard deviations, $\sigma$, on the components of $\mathbf{H}^{*}$ and $\mathbf{F}$ tensors calculated from ten different realizations of (a) the $W D$ unit cell and (b) the $S D$ unit cell. $\varepsilon=0.75, \nabla\left\langle p_{\beta}^{*}\right\rangle^{\beta}=\mathbf{e}_{\mathbf{x}}$. 
regime and validates the ensemble average approach used here.

\section{Non-Darcy correction}

Due to disorder and since solid inclusions are kept oriented, the angle $\theta_{v}$ that $\left\langle\mathbf{v}_{\beta}^{*}\right\rangle$ makes with $\nabla\left\langle p_{\beta}^{*}\right\rangle^{\beta}=\mathbf{e}_{\mathbf{x}}$ is expected to be zero for an infinite structure, whatever the Reynolds number, i.e., $\mathbf{H}^{*}$ and $\mathbf{F}$ are expected to be diagonal tensors. Numerical results on $\mathbf{K}^{*}$, above mentioned, show that this behavior is recovered on the average in the Darcy regime. Note that since $\nabla\left\langle p_{\beta}^{*}\right\rangle^{\beta}=\mathbf{e}_{\mathbf{x}}, \tan \left(\theta_{v}\right)=\frac{h_{y x}^{*}}{h_{x x}^{*}}\left(h_{i j}^{*}\right.$ is the $i j$ component of the $\mathbf{H}^{*}$ tensor) whereas $\mathbf{H}^{*} \rightarrow \mathbf{K}^{*}$ when $R e_{k} \rightarrow 0$. However, for each realization, this behavior is directly conditioned by the representative character of the periodic unit cell. When the Reynolds number increases, the amplitude of $\theta_{v}$ (i.e., the magnitude of the velocity component orthogonal to $\nabla\left\langle p_{\beta}^{*}\right\rangle^{\beta}$ relative to that along $\nabla\left\langle p_{\beta}^{*}\right\rangle^{\beta}$ ) is expected to decrease as a result of the modification of the flow structure within the medium. In fact, increasing the Reynolds number leads to an increasing channeling effect coupled to an increasing number of vortices that cancel out the average $y$ velocity component. This behavior suggested by Edwards et al. ${ }^{64}$ is confirmed for both $W D$ and $S D$ structures as shown in Figures 16(a) and 16(b) where the corre-

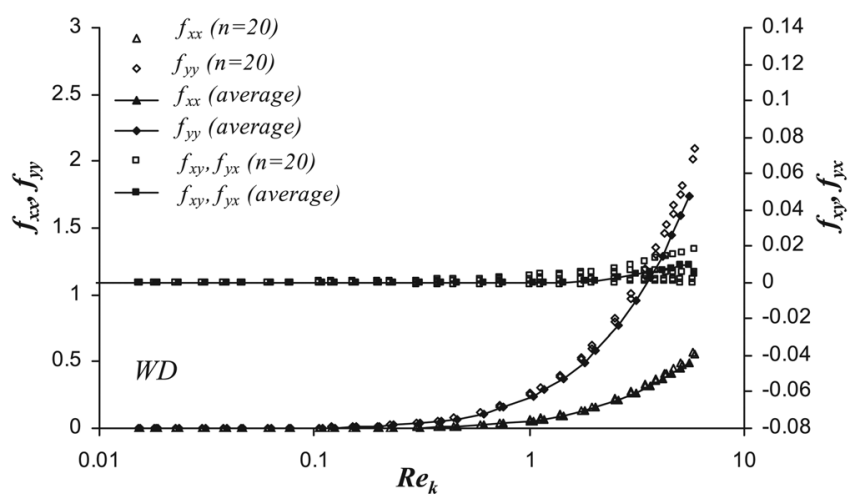

a)

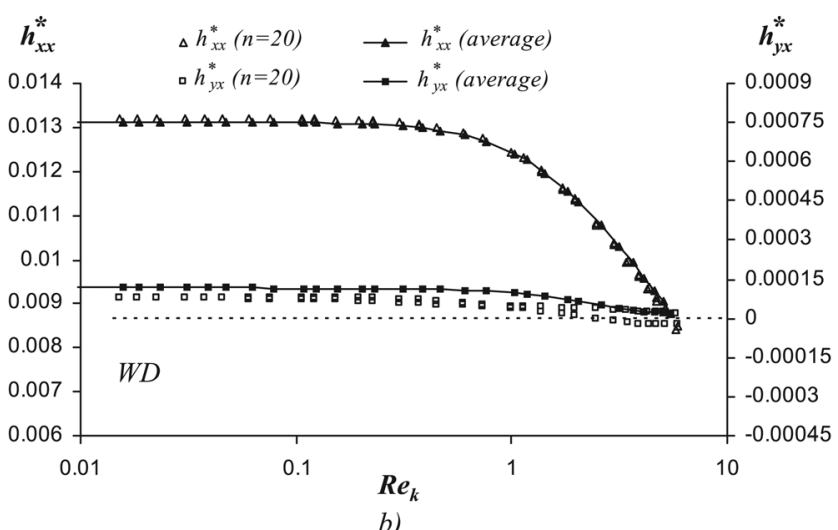

FIG. 19. Variation with $R e_{k}$ of the average values over ten realizations with $n=10$ and values of the same components for two realizations with $n=20$ of (a) the components of $\mathbf{F}$ and (b) $h_{x x}^{*}$ and $h_{y x}^{*}$. WD unit cell, $\varepsilon=0.75$, $\nabla\left\langle p_{\beta}^{*}\right\rangle^{\beta}=\mathbf{e}_{\mathbf{x}}$ sponding values of $\theta_{v}$ are reported versus $R e_{k}$. It can be clearly seen that $\theta_{v}$ is decreasing in magnitude for increasing $R e_{k}$ for all the realizations. Here, $R e_{k}$ was estimated with $\sqrt{\left(k_{x x}+k_{y y}\right) / 2}$ as the characteristic dimension.

As expected, $f_{x x}$ (and $f_{y y}$ ) are increasing with $R e_{k}$ (see Figures 17(a) and 17(b)). It must be noted, however, that offdiagonal terms of $\mathbf{F}$ are also significantly increasing in magnitude with $R e_{k}$ and this suggests to investigate more thoroughly the variation of the standard deviations, $\sigma$, of the $\mathbf{H}^{*}$ and $\mathbf{F}$ components with $R e_{k}$ represented in Figures 18(a) and $18(\mathrm{~b})$.

The dispersion on the two components $h_{x x}^{*}$ and $h_{y x}^{*}$ of interest decreases with $R e_{k}$, except that for $h_{x x}^{*}$ for the WD structure which remains quasi constant. However, the standard deviation on all the components of $\mathbf{F}$ are strongly increasing with $R e_{k}$. The latter behavior can be understood recalling the direct dependence of $\mathbf{F}$ upon $\mathbf{H}^{*-1}$ (see Eq. (35)) and the fact that components of $\mathbf{H}^{*}$ decrease with $R e_{k}$, along with the dispersion on $\mathbf{K}^{*}$. The important consequence of this is a decreasing (respectively increasing) size of the REV with respect to $\mathbf{H}^{*}$ (respectively $\mathbf{F}$ ) when $R e_{k}$ increases.

All the results observed on the average over the ten realizations are fully consistent with those obtained on two WD unit cells with $n=20$ and the same grid size (i.e., $800 \times 800$ grid blocks) (see Figures 16(a), 19(a), and 19(b)) corroborating again the validity of the ensemble average approach.
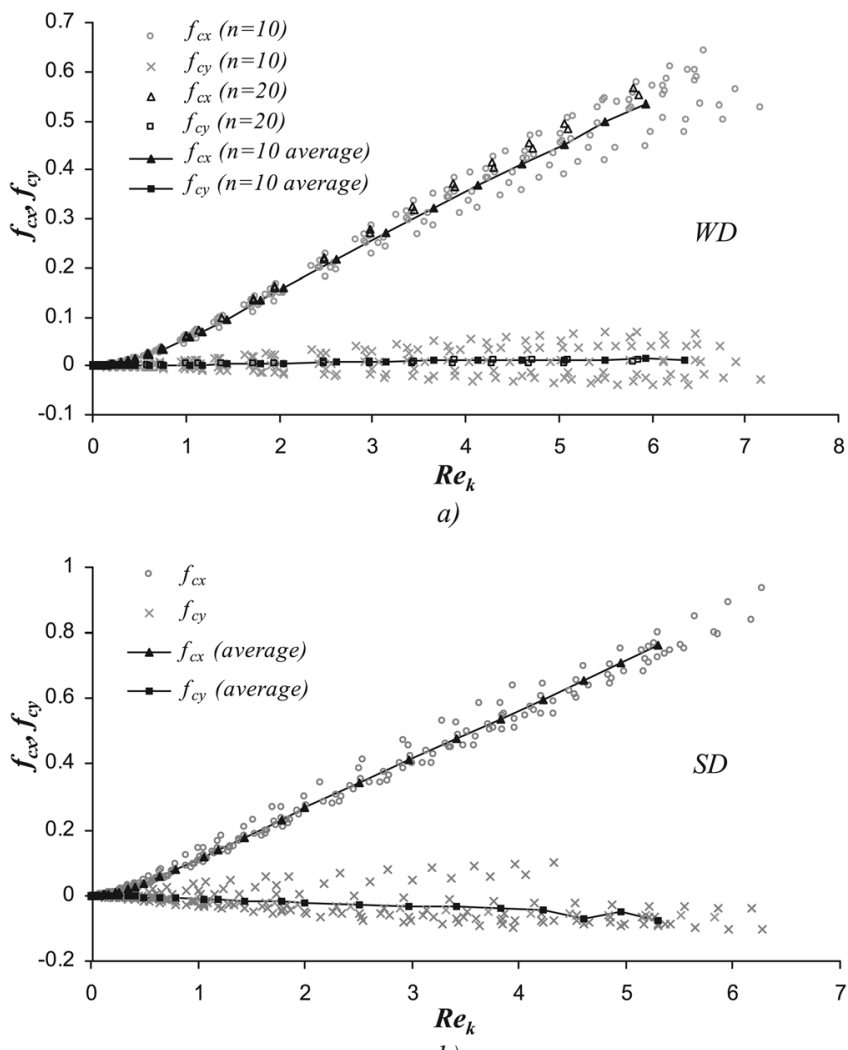

b)

FIG. 20. Variation of the components of $\mathbf{f}_{\mathbf{c}}$ with $R e_{k}$ for ten different realizations with $n=10$ and average of these values for (a) the WD unit cell; results for two realizations with $n=20$ are also reported and (b) the $S D$ unit cell. $\varepsilon=0.75, \nabla\left\langle p_{\beta}^{*}\right\rangle^{\beta}=\mathbf{e}_{\mathbf{x}}$ 


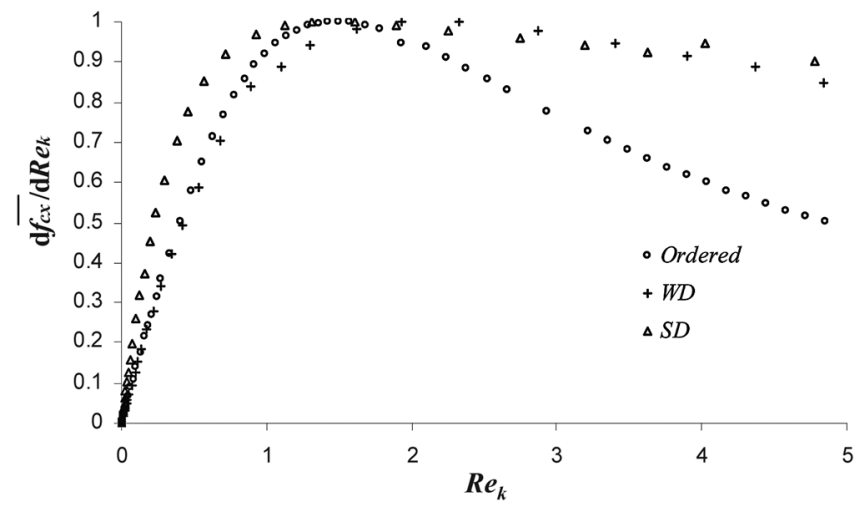

FIG. 21. Variation of $\frac{d \overline{f_{c x}}}{d R e_{k}}$ normalized by its maximum value versus $R e_{k}$ for the $W D$ and $S D$ structures. Comparison to $\frac{d f_{c x}}{d R e_{k}}$ obtained for the ordered structure with $\theta=0^{\circ}$ and $\varepsilon=0.75$.

Tests performed on one $W D$ structure with $n=10$ and $800 \times 800$ grid blocks indicate that the maximum relative error on $f_{x x}$ or $f_{y y}$ is less than $0.7 \%$ over the whole range of $R e_{k}$ confirming the very good accuracy of our results.

Because off-diagonal terms of $\mathbf{F}$ cancel over the investigated range of Reynolds numbers and since $\left\langle\mathbf{v}_{\beta}^{*}\right\rangle \cdot \mathbf{e}_{\mathbf{y}}$ is much smaller than $\left\langle\mathbf{v}_{\beta}^{*}\right\rangle \cdot \mathbf{e}_{\mathbf{x}}$ (at least 30 and 300 times for the $S D$ and $W D$ structures, respectively), the non-Darcy correction is investigated only while analyzing the dependence of $f_{c x}$ $\left(\simeq f_{x x}\right)$ on $R e_{k}$. A further justification of that is obvious from Figures 20(a) and 20(b) where the two components of $\mathbf{f}_{\mathbf{c}}$ are represented versus $R e_{k}$ for the $W D$ and $S D$ realizations.

Clearly, the correction $f_{c x}$ scales again as $R e_{k}^{2}$ at the onset of deviation from Darcy's law in the weak inertia regime that is followed by a transition regime. For larger Reynolds numbers, strong inertia regimes corresponding to excellent linear correlations with $R e_{k}$ are obtained. The range over which this correlation holds are very wide indicating that this approximation becomes much more precise and robust when disorder is introduced. This is illustrated in Figure 21 where $\frac{d \overline{c x}}{d R e_{k}}$, normalized by its respective maximum value, is represented versus $R e_{k}$ for $W D$ and $S D$ structures and compared to $\frac{d f_{c x}}{d R e_{k}}$ obtained for the ordered structure with $\theta=0^{\circ}$ and $\varepsilon=0.75$. This figure shows that $\frac{d \overline{c x}}{d R e_{k}}$ tends to a constant plateau for disordered media.
Coefficients $A, B, C$ and $D$ (see Eqs. (40) and (41)) as well as the crossover Reynolds numbers $R e_{k c}$ and $R e_{d c}$ defined in Eqs. (43) and (44) were determined on each realization. When possible (see discussion below), the Reynolds number ranges $\Delta R e_{k}$ (and $\Delta R e_{d}$ ) where the linear relationship $f_{c x}\left(R e_{k}\right)$ remains valid as well as the intervals $\delta R e_{k}$ of the transition between weak and strong inertia were also determined (see Sec. III A 1 for the definitions of both $\Delta R e_{k}$ and $\delta R e_{k}$ ). Averaged values of all these quantities (denoted with an over line) along with their corresponding standard deviations, $\sigma$, are reported in Table IV in which the same results for the ordered structure were recalled. These results call upon the following important conclusions. On all the realizations, $B$ is less than $10^{-5}$ confirming again the pure velocity cubic dependence of $\mathbf{f}_{\mathbf{i}}$ at the onset of deviation from Darcy's law. Values of $D$ are much larger in magnitude when disorder is introduced, leading to Forchheimer permeabilities markedly different from the intrinsic ones if a pure quadratic velocity dependence of $\mathbf{f}_{\mathbf{i}}$ is to be used in the strong inertia regime.

Moreover, intensity of inertial effects increases with disorder as indicated by the variations of $A$ and $C$ both being roughly one order of magnitude greater for the $S D$ structure in comparison to the ordered one. As in the ordered case for $\theta=0^{\circ}$, the non-Darcy correction remains extremely small compared to unity (around 0.03 at the crossover on the $S D$ structure) in the weak inertia regime. The decreasing value of $R e_{k c}$ (and $R e_{d c}$ ) with increasing disorder must also be emphasized. This effect is much more significant when strong disorder is introduced. One should note that for the $S D$ structure, $R e_{d c}$ is close to unity which is the usual admitted value at which Darcy regime is lost on real porous media. The Reynolds number ranges $\Delta R e_{k}$ and $\Delta R e_{d}$ corresponding to strong inertia increase significantly when disorder is introduced. In particular, for the $S D$ structure, $\Delta R e_{k}$ and $\Delta R e_{d}$ could not be estimated. Indeed, for the whole range of $R e_{k}$ over which computations were carried out (up to $R e_{k} \sim 6, R e_{d} \sim 24$ ), the linear relationship persists with less than $5 \%$ error. These results are a strong indication that the robustness of the quadratic dependence of the correction upon the velocity can be attributed to the disorder while dealing with natural porous structures. This sheds light on some observation recently made on numerical results obtained from pore-scale network modelling. ${ }^{76}$ This

TABLE IV. Average values and standard deviations of the coefficients in correlations (40) and (41), crossover Reynolds numbers and Reynolds number intervals corresponding to strong inertia $\left(\Delta R e_{k}, \Delta R e_{d}\right)$ and to the transition between weak and strong inertia $\left(\delta R e_{k}\right)$. Ordered, $W D$ and $S D$ structures. $\varepsilon=0.75$, $\nabla\left\langle p_{\beta}^{*}\right\rangle^{\beta}=\mathbf{e}_{\mathbf{x}}$.

\begin{tabular}{lccc}
\hline \hline Structure & Ordered & WD & SD \\
\hline $\bar{A}\left(\sigma_{A}\right)$ & $1.03 \times 10^{-2}$ & $7.0 \times 10^{-2}\left(6.4 \times 10^{-3}\right)$ & $0.20\left(4.9 \times 10^{-2}\right)$ \\
$\bar{B}\left(\sigma_{B}\right)$ & $1.3 \times 10^{-6}$ & $7.1 \times 10^{-6}\left(2.1 \times 10^{-6}\right)$ & $6.8 \times 10^{-6}\left(1.6 \times 10^{-6}\right)$ \\
$\bar{C}\left(\sigma_{C}\right)$ & $1.54 \times 10^{-2}$ & $0.10\left(8.0 \times 10^{-3}\right)$ & $0.15\left(1.2 \times 10^{-2}\right)$ \\
$\bar{D}\left(\sigma_{D}\right)$ & $-6.7 \times 10^{-3}$ & $-4.8 \times 10^{-2}\left(5.9 \times 10^{-3}\right)$ & $-4.1 \times 10^{-2}\left(6.6 \times 10^{-3}\right)$ \\
$\overline{R e_{k c}}\left(\sigma_{R e_{k c}}\right)$ & 0.74 & $0.73\left(6.6 \times 10^{-2}\right)$ & $0.40\left(6.8 \times 10^{-2}\right)$ \\
$\overline{R e_{d c}}\left(\sigma_{R e_{d c}}\right)$ & 3.25 & $3.19(0.28)$ & $1.57(0.27)$ \\
$\overline{\Delta R e_{k}}\left(\sigma_{\Delta R e_{k}}\right)$ & 2.31 & $5.34(0.66)$ & - \\
$\overline{\Delta R e_{d}}\left(\sigma_{\Delta R e_{d}}\right)$ & 10.11 & $23.37(2.98)$ & - \\
$\overline{\delta R e_{k}}\left(\sigma_{\delta R e_{k}}\right)$ & 0.59 & $0.96(0.03)$ & $0.52(0.13)$ \\
\hline \hline
\end{tabular}


also moderates and completes a conclusion put forth in the literature according to which the validity of the Forchheimer correction might be attributed to the 3D nature of the flow. ${ }^{27}$ Finally, we shall remark that the extent of the transition zone, $\delta R e_{k}$, between the weak and strong inertia regimes is not noticeably modified by the introduction of disorder.

\section{CONCLUSIONS}

Deviation from Darcy's law for one-phase incompressible flow in homogeneous porous media, when significant inertial effects are to be taken into account, was investigated. Our analysis is based on a macroscopic model valid in the framework of stationary flow over periodic structures. It includes a correction vector to Darcy's law involving a velocity dependent tensor $\mathbf{F}$. In the general case, $\mathbf{F}$ is given by an exact closure problem, the solution of which requires that of the microscopic flow. Both solutions can be obtained from the same Navier-Stokes solver.

Through comprehensive results obtained from extensive computations performed on model 2D structures, the different mean flow regimes have been analyzed versus the Reynolds number, pressure gradient orientation, and structural parameters such as porosity and disorder. These regimes were examined making use of a dimensionless form of the excess macroscopic force exerted on the structure relative to the flow in the Darcy regime. Major conclusions emerge from these results and can be listed as follows.

For ordered structures, in general, the correction tensor $\mathbf{F}$ is a dense non-symmetric tensor even if the structure is macroscopically isotropic in the Darcy regime. This is in accordance with the fact that the force is not pure drag, i.e., is not aligned with the mean flow. The exception is when the applied pressure gradient is along a symmetry axis of the representative unit cell. For disordered isotropic media, F should be diagonal when estimated over a Representative Elementary Volume, the size of which increases with increasing Reynolds numbers.

Intensity of inertial effects always increases with increasing Reynolds numbers. Special attention must be however dedicated to the definition of the Reynolds number. For an isotropic ordered structure, intensity of inertial effects is a decreasing function of the porosity when the Reynolds number is defined with the square root of the permeability. The opposite holds when the Reynolds number is defined in the classical manner using the grain size as the characteristic length.

In all cases studied here, a weak inertia regime characterized by a square dependence of the correction on the Reynolds number, i.e., a cubic dependence on the velocity, is always recovered at the onset of deviation from Darcy's law in accordance with theoretical results reported in the literature. In most cases under study, the intensity of the correction in this regime is insignificant, except on ordered structures with particular pressure gradient orientations. This last situation is however a realistic one for many applications where synthetic highly ordered structures are of particular interest like for instance in MEMS, micro heat exchangers, etc.

For higher Reynolds numbers, the strong inertia regime where the dimensionless correction is expected to linearly depend upon the Reynolds number (i.e., a quadratic dependence of its dimensional form on the velocity) is a more or less robust approximation. For ordered structures, it only holds on an interval of Reynolds numbers of variable restricted extent centered on an inflexion point of the correction as a function of the Reynolds number. Moreover, since it is not purely quadratic, this would lead to the introduction of a so-called "Forchheimer permeability" distinct from the intrinsic one if a Forchheimer correction is to be used instead. For our ordered model structure, the strong inertia regime is never observed for a pressure gradient orientation of $45^{\circ}$ with respect to the axes of the structure.

A crossover Reynolds number, minimizing the difference between the weak and strong inertia models, was introduced. When based on the square root of the permeability, the crossover Reynolds number is an increasing function of the porosity while the opposite holds when the definition of the Reynolds number is based on the grain size. This crossover Reynolds number significantly decreases when strong disorder is introduced. The extent of the transition zone is not markedly affected by disorder.

On disordered structures, the velocity quadratic dependence of the correction is a robust approximation in a very large interval of Reynolds numbers. However, the permeability significantly differs from the intrinsic one if a Forchheimer correction is used. In addition, the crossover Reynolds number and the magnitude of the weak inertia correction are both small explaining why this regime is generally overlooked during experiments. Finally, it should be noticed that preliminary calculations carried out on one realization of the $S D$ structure for $\theta=30^{\circ}$ and $R e_{k}$ up to 8 indicate that $f_{x y}-f_{y x}$, that measures the dissymmetry of the $\mathbf{F}$ tensor, is always smaller than $f_{x y}-f_{y x}$ for the ordered structure. The contrast reaches one order of magnitude at $R e_{k} \sim 8$. Similarly, $\theta_{v}$ is also very significantly reduced (by a factor $\sim 3$ at this particular value of $R e_{k}$ ) while introducing disorder. More results are required to thoroughly investigate this behavior that was beyond the scope of the present work.

Although disorder was investigated in two dimensions, these results provide indications that the same conclusions might be safely extended to $3 \mathrm{D}$ real porous structures with even intensified expected effects in that case. Further work can now be planned on anisotropic media and, on the basis of a second upscaling, on heterogeneous structures.

\section{APPENDIX A: VALIDATION}

The objective of this appendix is to compare our numerical results on the permeability and non-Darcy correction with existing data reported in the literature and with results obtained with a Boundary Element Method (BEM). The comparison is performed using the model configuration of Figure 1(a) for which the characteristics are given in Sec. II C.

\section{Permeability}

Although some relatively precise estimates of $k^{*}$ are available in this case, at least at large values of the porosity, ${ }^{77}$ no exact analytical results for the flow are available on 
such a structure and comparison can only be performed with other numerical results. The Stokes flow was computed with the finite volume (FV) method detailed in Sec. II C. The convergence criterion of the overall algorithm was $10^{-12}$ on the $L^{2}$ norm of the divergence of the velocity over all grid blocks while the relative error criterion for the linear system solver was $10^{-15}$. The problem was also solved with a BEM using constant elements which reveals to be very precise for this type of problem. ${ }^{78}$ All the results on $k^{*}$ are gathered in Table V.

Although the numerical method of the present work seems to very slightly underestimate the permeability in this particular configuration, results are in very good agreement since the maximum relative error between all these values is less than $3.6 \%$, taking the result obtained with the BEM as the reference value.

\section{Non-Darcy correction}

The dependence of $f_{c x}=f_{x x}$ on $R e_{k}$ corresponding to $R e_{d}$ ranging from 0 to 60 is compared to results reported in Ref. 27 and is represented in Figure 22.

The agreement is excellent leading to a relative difference always less than $0.5 \%$ over the whole range of $R e_{k}$ which validates the present numerical method. Truncations on either $\varepsilon$ or $d / l$ are probably not identical in the present work and in the cited reference and this can possibly be part of the explanation of this small difference.

\section{APPENDIX B: CORRELATIONS WITH POROSITY}

In this appendix, correlations between the porosity $\varepsilon$ and different characteristics $\left(k^{*}, \beta, R e_{k c}, R e_{d c}\right.$, and $\left.\Delta R e_{k}\right)$ deduced from our numerical results obtained on the ordered structure of Sec. III B are discussed.

\section{1. $\mathbf{k}^{*}-\varepsilon$ correlation}

To account for the dependence of $k^{*}$ on $\varepsilon$ over the whole range of porosity, it is convenient to use a relationship of the form. ${ }^{9}$

$$
a \varepsilon^{\alpha_{1}}(1-\varepsilon)^{\alpha_{2}}
$$

rather than employing estimates from approximated solutions to the flow that are asymptotically valid at exceedingly small or large porosities. A least square fit of the form (B1) performed on $k^{*}$ yields

TABLE V. Permeability results obtained with the finite volumes method (FV) developed in this work. Comparison to BEM and existing results. ${ }^{27}$ Unit cell of Figure 1(a), $\varepsilon=0.386$.

\begin{tabular}{lc}
\hline \hline Method (\#of grid blocks) & $k^{*}$ \\
\hline FEMLAB $^{27}$ (unknown) & $2.3111 \times 10^{-4}$ \\
BEM $(14700$ elements) & $2.2952 \times 10^{-4}$ \\
FV $(500 \times 500)$ & $2.2130 \times 10^{-4}$ \\
FV $(1000 \times 1000)$ & $2.2505 \times 10^{-4}$ \\
FV $(1500 \times 1500)$ & $2.2663 \times 10^{-4}$ \\
\hline \hline
\end{tabular}

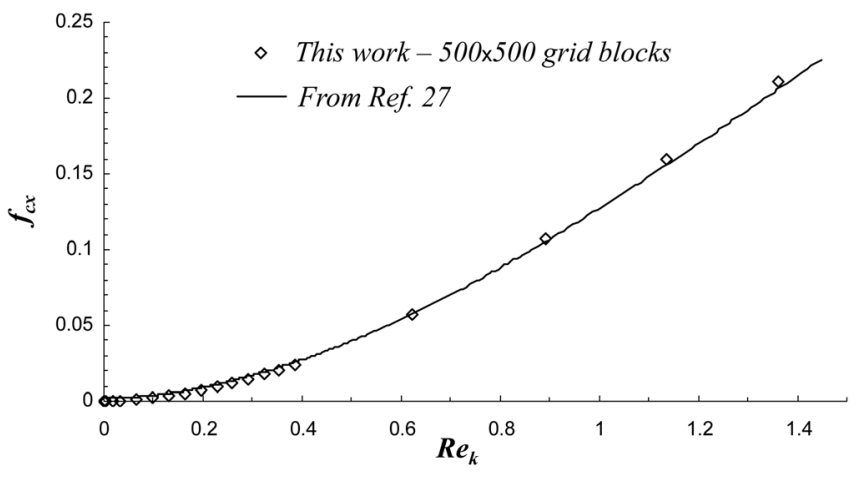

FIG. 22. Variation of $f_{c x}$ versus $R e_{k}$ for the unit cell of the model configuration in Figure 1(a). Comparison with existing results. ${ }^{27}$

$$
\left\{\begin{array}{c}
k^{*} \simeq 0.015 \frac{\varepsilon^{3.23}}{(1-\varepsilon)^{0.57}} \quad 0.3 \lesssim \varepsilon \lesssim 0.75 \\
E r r \simeq 510^{-11}
\end{array}\right.
$$

where $E r r$ is the mean square error of the estimator of $k^{*}$.

In Eq. (B2), exponents are slightly different from those in the Kozeny relationship originally derived for sphere packs but extensively used for a wide variety of unconsolidated structures $9,13,50$ and in which exponents would be $\alpha_{1}=3$ and $\alpha_{2}=-1$. The pre-factor $a=0.015$ in equation (B2) is also different from the value 0.00667 proposed by Ergun or the value 0.00556 deduced from fits on experimental data by MacDonald et al. ${ }^{13}$ over a wide variety of media and used later by Amaral Souto and Moyne. ${ }^{50}$ However, as already pointed out, sensitivity to errors on coefficients appearing in the drag coefficient to Reynolds number relationship is weak to precisely estimates these coefficients. One of the conclusions from MacDonald et al. ${ }^{13}$ is that their proposed fit would be precise to within $\pm 50 \%$.

\section{2. $\beta-\varepsilon$ correlation}

If the strong inertia regime is assumed to be described by a Forchheimer model, implying no weak inertia regime and $D=0$ in the relationship (41), the inertial resistance factor $\beta$ (see Equation (3)) is related to $\mathrm{C}$ by $\frac{C}{\sqrt{k}}=\beta$, i.e., $\frac{C}{\sqrt{k^{*}}}=\beta l$. Carrying out a least square fit of the form (B1) on the values of $\frac{C}{\sqrt{k^{*}}}$ obtained on the ordered structure under study yields

$$
\left\{\begin{array}{c}
\frac{C}{\sqrt{k^{*}}} \simeq 0.12 \frac{(1-\varepsilon)^{0.38}}{\varepsilon^{2.04}} \quad 0.3 \lesssim \varepsilon \lesssim 0.75 \\
E r r \simeq 1.110^{-5}
\end{array},\right.
$$

where Err is again the mean square error of the estimator of $\frac{C}{\sqrt{k^{*}}}$. Exponents and pre-factor obtained from this fit are also slightly different from those appearing in widely used correlations proposed by Ergun ${ }^{9}$ based on an implicit model of packed spheres where $\alpha_{1}=-3, \alpha_{2}=0.5, a=1.75$ or by MacDonald et $a .^{13}$ and used by Amaral Souto and Moyne ${ }^{50}$ where $\alpha_{1}=-3, \alpha_{2}=0.5, a=1.8$. Discrepancy of these relationships with computed results obtained on square and hexagonal arrays of uniform and non uniform cylinders of circular cross section was examined by Papathanasiou 
et $a l .{ }^{26}$ through the analysis again of the relationship between the drag coefficient and the Reynolds number. Using $R e_{k}$ in the expression of the drag coefficient to advantageously avoid an implicit relationship between $k$ and any other structural dimension, they concluded to a relationship implying, with our notations, $C \simeq 0.08 \frac{1-\varepsilon}{\varepsilon}$. Although the discrepancy of this relation with our results must be found in the difference of cylinder shape, special attention must be paid to the accuracy of such a correlation when extracted from the drag coefficient to Reynolds number relationship.

\section{3. $\mathrm{Re}_{\mathrm{kc}}-\varepsilon, \mathrm{Re}_{\mathrm{dc}}-\varepsilon$ and $\Delta \mathrm{Re}_{\mathrm{k}}-\varepsilon$ correlations}

As for $k^{*}$ and $C$, it is appealing to correlate $R e_{k c}, R e_{d c}$ and $\Delta R e_{k}$ to $\varepsilon$ in the form reported in Eq. (B1) where porosity limits are involved. For the structure under concern and the range of porosity investigated here, we find

$$
\begin{gathered}
\left\{\begin{array}{c}
R e_{k c} \simeq 0.69 \frac{\varepsilon^{1.49}}{(1-\varepsilon)^{0.36}} \quad 0.3 \lesssim \varepsilon \lesssim 0.75 \\
E r r \simeq 1.010^{-5}
\end{array},\right. \\
\left\{\begin{array}{c}
R e_{d c} \simeq 6.96 \varepsilon^{0.05}(1-\varepsilon)^{0.55} \quad 0.3 \lesssim \varepsilon \lesssim 0.75 \\
E r r \simeq 1.410^{-3}
\end{array}\right.
\end{gathered}
$$

and

$$
\left\{\begin{array}{c}
\Delta R e_{k} \simeq 4.09 \varepsilon^{1.94}(1-\varepsilon)^{0.01} 0.3 \lesssim \varepsilon \lesssim 0.75 \\
E r r \simeq 1.610^{-4}
\end{array} .\right.
$$

As shown in Eqs. (B5) and (B6), $R e_{d c}$ has a quasi squareroot dependence on $(1-\varepsilon)$ while $\Delta R e_{k}$ mainly depends on the square of $\varepsilon$.

${ }^{1}$ H. Darcy, "Fontaines publiques de la ville de Dijon,"Librairie des Corps Impériaux des Ponts et Chaussées et des Mines, Paris (1856).

${ }^{2}$ E. Sanchez-Palencia, Non Homogeneous Media and Vibration Theory, Lecture Notes in Physics (Springer, Berlin, Germany, 1980).

${ }^{3} \mathrm{~S}$. Whitaker, "Flow in porous media i: A theoretical derivation of Darcy's law," Transp. Porous Media 1, 3 (1986).

${ }^{4}$ P. Forchheimer, "Wasserbewegung durch boden," Z. Ver. Dtsch. Ing. XXXXV, 1781 (1901).

${ }^{5} \mathrm{G}$. Chauveteau, "Essai sur la loi de Darcy et Les écoulements laminaires à Perte de charge non linéaire," Ph.D. thesis (University of Toulouse, 1965).

${ }^{6}$ L. Green and P. Duwez, "Fluid flow through porous metals," J. Appl. Mech. 18, 39 (1951).

${ }^{7}$ D. Cornell and L. K. Katz, "Flow of gases through consolidated porous media," Ind. Eng. Chem. 45, 2145 (1953).

${ }^{8} \mathrm{G}$. Schneebeli, "Expériences sur la limite de validité de la loi de Darcy et l'apparition de la turbulence dans un écoulement de filtration," La Houille Blanche 2, 141 (1955).

${ }^{9}$ S. Ergun, "Fluid flow through packed columns," Chem. Eng. Prog. 48, 89 (1952).

${ }^{10}$ J. C. Ward, "Turbulent flow in porous media," J. Hyd. Div., ASCE 90, 1 (1964).

${ }^{11}$ G. S. Beavers and E. M. Sparrow, "Non-Darcy flow through fibrous porous media," Trans. ASME J. Appl. Mech. December, 711 (1969).

${ }^{12}$ F. A. L. Dullien and M. I. S. Azzam, "Flow rate-pressure gradient measurement in periodically nonuniform capillary tubes," AIChE J. 19, 222 (1973).

${ }^{13}$ I. F. MacDonald, M. S. El-Sayed, K. Mow, and F. A. L. Dullien, "Flow through porous media-the Ergun equation revisited," Ind. Eng. Chem. Fundam. 18, 199-208 (1979).

${ }^{14} \mathrm{~S}$. Irmay, "On the theoretical derivation of Darcy and Forchheimer formulas," J. Geophys. Res. 39, 702 (1958).

${ }^{15}$ E. F. Blick, "Capillary orifice model for high speed flow through porous media,” I\&EC, Proc. Des. Dev. 1, 90 (1966).
${ }^{16}$ N. Ahmed and D. K. Sunada, "Nonlinear flow in porous media," J. Hydr. Div. ASCE 95, 1847 (1969).

${ }^{17}$ V. D. Cvetkovic, "A continuum approach to high velocity flow in a porous medium," Transp. Porous Media 1, 63 (1986).

${ }^{18}$ S. M. Hassanizadeh and W. G. Gray, "High velocity flow in porous media," Transp. Porous Media 2, 521 (1987).

${ }^{19} \mathrm{D}$. Ruth and H. Ma., "On the derivation of the Forchheimer equation by means of the averaging theorem," Transp. Porous Media 7, 255 (1993a)

${ }^{20}$ T. Giorgi, "Derivation of the Forchheimer law via matched asymptotic expansions," Transp. Porous Media 29, 191 (1997).

${ }^{21}$ Z. Chen, S. L. Lyons, and G. Qin, "Derivation of the Forchheimer law via homogenization," Transp. Porous Media 44, 325 (2001).

${ }^{22}$ O. Coulaud, P. Morel, and J. P. Catagirone, "Numerical modeling of nonlinear effects in laminar flow through a porous medium," J. Fluid Mech. 190, 393 (1988).

${ }^{23} \mathrm{H}$. Ma and D. W. Ruth, "The microscopic analysis of high Forchheimer number flow in porous media," Transp. Porous Media 13, 139 (1993).

${ }^{24} \mathrm{D}$. Ruth and H. Ma., "Numerical analysis of the viscous incompressible flow in a diverging-converging RUC," Transp. Porous Media 13, 161 (1993b).

${ }^{25} \mathrm{~F}$. Thauvin and K. K. Mohanty, "Network modeling of non-Darcy flow through porous media," Transp. Porous Media 31, 19 (1998).

${ }^{26}$ T. D. Papathanasiou, B. Markicevic, and E. D. Dendy, "A computational evaluation of the Ergun and Forchheimer equations for fibrous porous media," Phys. Fluids 13, 2795 (2001).

${ }^{27}$ M. Fourar, G. Radilla, R. Lenormand, and C. Moyne, "On the non-linear behavior of a laminar single-phase flow through two and three-dimensional porous media," Adv. Water Resour. 27, 669 (2004).

${ }^{28}$ A. R. Mazaheri, B. Zerai, G. Ahmadi, J. R. Kadambi, B. Z. Saylor, M. Oliver, G. S. Bromhal, and D. H. Smith, "Computer simulation of flow through a lattice flow-cell model," Adv. Water Resour. 28, 1267 (2005).

${ }^{29}$ C. Garibotti and M. Peszyńska, "Upscaling non-Darcy flow," Transp. Porous Media 80, 401 (2009).

${ }^{30} \mathrm{~J}$. Bear, Dynamics of Fluids in Porous Media (Dover, New York, 1972).

${ }^{31}$ A. E. Scheidegger, The Physics of Flow Through Porous Media (University of Toronto Press, Toronto, Canada, 1974).

${ }^{32} \mathrm{~J}$. Geertsma, "Estimating the coefficient of inertial resistance in fluid flow through porous media," Soc. Pet. Eng. J., 14, 445 (1974).

${ }^{33}$ M. R. Tek, "Development of a generalized Darcy equation," J. Petrol. Technol. 9, 45 (1957).

${ }^{34}$ D. E. Wright, "Nonlinear flow through granular media," J. Hyd. Div. ASCE 4, 851 (1968).

${ }^{35}$ A. Dybbs and R. V. Edwards, "A new look at porous media fluid mechanics-darcy to turbulent," in Fundamentals of Transport Processes in Porous Media, edited by J. Bear and M. Y. Corapcioglu (Nijhoff, Dordrecht, 1984), pp. 201-256.

${ }^{36}$ M. R. Tek, K. H. Coats, and D. L. Katz, "The effect of turbulence on flow of natural gas through porous reservoirs," J. Petrol. Technol. 14, 799 (1962).

${ }^{37} \mathrm{G}$. Chauveteau and C. Thirriot, "Régimes d'écoulement en milieu poreux et limite de la loi de darcy," La Houille Blanche 2, 141 (1967).

${ }^{38} \mathrm{C}$. K. Ghaddar, "On the permeability of unidirectional fibrous media: A parallel computational approach,” Phys. Fluids 7, 2563 (1995).

${ }^{39}$ J. B. Koch and J. C. Ladd, "Moderate Reynolds number flows through periodic and random arrays of aligned cylinders," J. Fluid Mech. 340, 31 (1997).

${ }^{40}$ J. Barrère, "Modélisation des Écoulement de Stokes et de Navier-Stokes en Milieu Poreux," Ph.D. thesis (University of Bordeaux, 1990).

${ }^{41}$ J.-C. Wodie and T. Levy, "Correction non linéaire de la loi de Darcy," C. R. Acad. Sci. Paris II 312, 157 (1991).

${ }^{42}$ C. C. Mei and J. L. Auriault, "The effect of weak inertia on flow through a porous medium,” J. Fluid Mech. 222, 647 (1991).

${ }^{43}$ M. Rasoloarijaona and J. L. Auriault, "Nonlinear seepage flow through a rigid porous medium," Eur. J. Mech. B/Fluids 13, 177 (1994).

${ }^{44}$ E. Skjetne and J. L. Auriault, "High-velocity laminar and turbulent flow in porous media," Transp. Porous Media 36, 131 (1999a).

${ }^{45}$ M. Balhoff, A. Mikelic, and M. Wheeler, "Polynomial filtration laws for low Reynolds number flows through porous media," Transp. Porous Media 81, 35 (2010).

${ }^{46} \mathrm{M}$. Firdaouss and J. Guermond, "Sur l'homogénéisation des équations de Navier-Stokes à faible nombre de Reynolds," C. R. Acad. Sci. Paris, Série I 320, 245 (1995). 
${ }^{47}$ M. Firdaouss, J. L. Guermond, and P. Le Quéré, "Nonlinear corrections to Darcy's law at low Reynolds numbers," J. Fluid Mech. 343, 331 (1997).

${ }^{48}$ E. Skjetne and J. L. Auriault, "New insights on steady, non-linear flow in porous media," Eur. J. Mech. B/Fluids 18, 131 (1999b).

${ }^{49}$ D. Lo Jacono, F. Plouraboué, and A. Bergeon, "Weak inertial flow between two rough surfaces," Phys. Fluids 17, 063602 (2005).

${ }^{50} \mathrm{H}$. P. Amaral Souto and C. Moyne, "Dispersion in two-dimensional periodic porous media, part i. hydrodynamics," Phys. Fluids 9, 2243 (1997).

${ }^{51}$ S. Rojas and J. Koplik, "Nonlinear flow in porous media," Phys. Rev. E 58, 4776 (1998).

${ }^{52}$ E. Skjetne, A. Hansen, and J. S. Gudmundsson, "High velocity flow in a rough fracture," J. Fluid Mech. 383, 1 (1999).

${ }^{53}$ S. Whitaker, "The Forchheimer equation: A theoretical development," Transp. Porous Media 25, 27 (1996).

${ }^{54} \mathrm{~J}$. Happel and B. Brenner, Low Reynolds Number Hydrodynamics (Prentice-Hall, Englewood Cliffs, NJ., 1965).

${ }^{55}$ T. Suekane, Y. Yokouchi, and S. Hirai, "Inertial flow structures in a simple-packed bed of spheres," AIChE J. 49, 10 (2003).

${ }^{56}$ R. E. Hayes, A. Afacan, and B. Boulanger, "An equation of motion for an incompressible Newtonian fluid in a packed bed," Transp. Porous Media 18, 185 (1995).

${ }^{57}$ J. S. Andrade, U. M. S. Costa, M. P. Almeida, H. A. Makse, and H. E. Stanley, "Inertial effects on fluid flow through disordered porous media," Phys. Rev. Lett. 82, 5249 (1999).

${ }^{58}$ M. Panfilov and M. Fourar, "Physical splitting of non linear effects in high-velocity stable flow through porous media," Adv. Water Resourc. 29, 30 (2006)

${ }^{59}$ A. Barak and J. Bear, "Flow at high Reynolds numbers through anisotropic porous media," Adv. Water Resourc. 4, 54 (1981).

${ }^{60}$ D. Lasseux, A. Ahmadi, and A. Abbasian Arani, "Two-phase inertial flow in homogeneous porous media: A theoretical derivation of a macroscopic model," Transp. Porous Media 75, 371 (2008).

${ }^{61}$ A. Ahmadi, A. Abbasian Arani, and D. Lasseux, "Numerical simulation of two-phase inertial flow in heterogeneous porous media," Transp. Porous Media 84, 177 (2010).

${ }^{62}$ J. Barrère, O. Gipouloux, and S. Whitaker, "On the closure problem for Darcy's law,” Transp. Porous Media 7, 209 (1992).

${ }^{63}$ S. Whitaker, Theory and Applications of Transport in Porous Media: The Method of Volume Averaging (Kluwer Academic, Dordrecht, The Netherlands, 1999).
${ }^{64}$ D. A. Edwards, M. Shapiro, P. Bar-Yoseph, and M. Shapira, "The influence of Reynolds number upon the apparent permeability of spatially periodic arrays of cylinders," Phys. Fluids 2, 45 (1990).

${ }^{65}$ H. I. Ene and E. Sanchez-Palencia, "Equations et phénomènes de surface pour l'écoulement dans un modèle de milieu poreux," J. Méc. 14, 73 (1975).

${ }^{66}$ W. G. Gray, "A derivation of the equations for multiphase transport," Chem. Eng. Sci. 30, 229 (1975).

${ }^{67}$ M. Zuzovsky, P. M. Adler, and H. Brenner, "Spatially periodic suspensions of convex particles in linear shear flows. III. dilute arrays of spheres suspended in Newtonian fluids," Phys. Fluids 26, 1714 (1983).

${ }^{68}$ S. V. Patankar, Numerical Heat Transfer and Fluid Flow (Hemisphere Pub. Corp., Washington, 1980).

${ }^{69}$ B. P. Leonard, "A stable and accurate convective modelling procedure based on quadratic upstream interpolation," Comput. Methods Appl. Mech. Eng. 19, 59 (1979).

${ }^{70}$ M. A. Leschziner, "Practical evaluation of three finite difference schemes for the computation of steady-state recirculating flows," Comput. Methods Appl. Mech. Eng. 23, 293 (1980).

${ }^{71}$ T. Han, J. A. C. Humphrey, and B. E. Launder, "A comparison of hybrid and quadratic-upstream differencing in high Reynolds number elliptic flows," Comput. Methods Appl. Mech. Eng. 29, 81 (1981).

${ }^{72}$ A. Pollard and A. L. W. Siu, "The calculation of some laminar flows using various discretisation schemes," Comput. Methods Appl. Mech. Eng. 35, 293 (1982).

${ }^{73}$ C. J. Freitas, R. L. Street, A. N. Findikakis, and J. R. Koseff, "Numerical simulation of three-dimensional flow in cavity," Int. J. Numer. Methods Fluids 5, 561 (1985).

${ }^{74}$ T. Hayase, J. A. C. Humphrey, and R. Greif, "A consistently formulated QUICK scheme for fast and stable convergence using finite-volume iterative calculation procedures," J. Comput. Phys. 98, 108 (1992).

${ }^{75}$ R. Peyret and T. D. Taylor, Computational Methods for Fluid Flow (Springer-Verlag, New York, 1983).

${ }^{76} \mathrm{M}$. Balhoff and $\mathrm{M}$. Wheeler, "A predictive pore-scale model for nonDarcy flow in porous media,” SPE J. 4, 579-587 (2009).

${ }^{77}$ J. E. Drumond and M. I. Tahir, "Laminar viscous flow through regular arrays of parallel solid cylinders," Int. J. Multiphase Flow 10, 515 (1984).

${ }^{78}$ R. E. Larson and J. J. L. Higdon, "Microscopic flow near the surface of two-dimensional porous media. part 2. transverse flow," J. Fluid Mech. 178, 119 (1987). 